\title{
Awake responses suggest inefficient dense coding in the mouse retina
}

Authors: Tom Boissonnet ${ }^{1,2}$, Matteo Tripodi ${ }^{1}$, Hiroki Asari ${ }^{\star 1}$

\section{Affiliations:}

1. Epigenetics and Neurobiology Unit, EMBL Rome, European Molecular Biology Laboratory, Monterotondo, 00015, Italy

2. Collaboration for joint PhD degree between EMBL and Université Grenoble Alpes, Grenoble Institut des Neurosciences, La Tronche, 38700, France

*Corresponding author: Hiroki Asari, E-mail: asari@embl.it

Author contributions: T.B. and H.A. designed the study; T.B. and M.T. performed experiments; T.B. analyzed the results; and T.B. and H.A. wrote the manuscript.

Competing interests: The authors declare no competing financial interests.

Key words: retinal ganglion cells; in vivo recordings; awake; anesthesia; efficient coding.

Acronyms: CMOS, complementary metal-oxide semiconductor; DS, direction-selectivity; dLGN, dorsal lateral geniculate nucleus; DLP, digital light processing; FMM, fentanyl, medetomidine and midazolam; GABA, gamma-aminobutyric acid; LED, light emitting diode; OS, orientation-selectivity; OT, optic tract; PCA, principal component analysis; PFA, paraformaldehyde; RGC, retinal ganglion cells; SC, superior colliculus; STA, spike-triggered average; UV, ultraviolet. 


\section{Abstract}

2 The structure and function of the vertebrate retina have been extensively studied across species with an isolated, ex vivo preparation. Retinal function in vivo, however, remains elusive, especially in awake animals. Here we performed single-unit extracellular recordings in the optic tract of head-fixed mice to compare the output of awake, anesthetized, and ex vivo retinas. While the visual response properties were overall similar, we found that awake retinal output had 1) faster kinetics with less variability in the response latencies across different cell types; and 2) higher firing activity, by $\sim 20 \mathrm{~Hz}$ on average, for both baseline and visually evoked responses. Notably, unlike the other conditions, many awake ON cells did not increase firing in response to light increments due to high baseline activity near saturation. Instead, they encoded light intensity fluctuations primarily by decreasing firing upon light decrements. In either condition, the visual message remains the same: the more spikes, the higher light intensity. The awake response patterns, however, violate efficient coding principles, predicting that sensory systems should favor firing patterns minimizing energy consumption. Our findings suggest that the retina employs dense coding in vivo, rather than sparse efficient coding as suggested from previous ex vivo studies.

\section{Introduction}

The vertebrate retina is one of the best characterized parts of the central nervous system (Gollisch and Meister, 2010; Masland, 2012). It consists of $\sim 100$ cell types in total (Shekhar et al., 2016; Yan et al., 2020), including 30 types of retinal ganglion cells (RGCs) that send the retinal output signals to the brain via the optic nerve (Sanes and Masland, 2015; Baden et al., 2016). Each of these RGC types forms distinct neural circuits within the retina to extract specific features of the visual image coming into the eye, such as color, contrast, and motion. The retina thus performs parallel and dynamic processing as the first stage of the visual pathway. 
Most of our knowledge on retinal function has been obtained from ex vivo studies because isolated retinal tissues are nevertheless functional, i.e., responsive to light (Barlow et al., 1964). While powerful, however, ex vivo physiological approaches have certain limitations. First, one cannot perform long-term recordings (Meister et al., 1994). Second, one cannot avoid artifacts due to retinal dissection, such as the effect of cutting RGC axons (Vidal-Sanz et al., 2017) or retinal epithelial detachment (Strauss, 2005). Neuromodulatory effects of retinopetal pathways are also difficult to study in an isolated retinal preparation (Repérant et al., 2006; Esposti et al., 2013). In vivo studies are thus indispensable for clarifying retinal function thoroughly.

Previous physiological studies on the retina in vivo were conducted mostly under anesthetized—and often paralyzed—conditions. To monitor RGC activity in vivo, for example, single-unit recordings were made directly from the retina (Kuffler, 1953), at the optic nerve or tract fibers (Hartline, 1938; Lettvin et al., 1959; Enroth-Cugell and Robson, 1966; Mastronarde, 1983, 1985; Sagdullaev and McCall, 2005), or in the form of "slow-potential" in the dorsal lateral geniculate nucleus (dLGN; Bishop et al., 1962; Kaplan and Shapley, 1984). Optical methods were also developed to image the activity of retinal neurons directly through the pupil of a live animal (Geng et al., 2012; Yin et al., 2013, 2014). In contrast, thus far only a handful of studies have reported awake recordings from the retina (Esposti et al., 2013; Hong et al., 2018) or its outputs (Weyand, 2007; Liang et al., 2018, 2020; Schröder et al., 2020; Sibille et al., 2021). Thus, despite a long history of research on the retina, it still remains unclear what exactly the eye tells the brain in awake animals.

As recordings from awake behaving animals became routine for many brain areas (Dombeck et al., 2007; Jun et al., 2017), growing attention has been paid to the roles of an animal's behavior and internal brain states in the function of the sensory systems (Niell and Stryker, 2010; Lee and Dan, 2012). For example, systematic studies on the early visual pathway showed higher firing activity and faster response dynamics in both dLGN (Durand et al., 2016) and the superior colliculus (SC; De Franceschi and Solomon, 2018) of awake 
animals than those under anesthesia. A critical question that was left unanswered is to what extent such differences originate in the retina.

To clarify differences in the retinal visual response properties between awake and anesthetized conditions, here we employed single-unit extracellular recording techniques from head-fixed mice. Specifically, the electrodes were placed in the optic tract (OT), a bundle of nerve fibers composed of RGC axons as they project from the eye to their main targets: dLGN and SC. These recordings are superior to direct in vivo retinal recordings (e.g., with epiretinally implanted mesh electrodes; Hong et al., 2018) because the retinal circuits and the eye optics remain intact, and also to those extracellular recordings in SC (Sibille et al., 2021) or dLGN (Weyand, 2007) because RGC axonal signals do not need to be disambiguated from those of local axons or somata. This advantage also exists for calcium imaging recordings of the retina directly in the eye of immobilized zebrafish larvae (Esposti et al., 2013) or those of RGC axon terminals in dLGN (Liang et al., 2018, 2020) or SC (Schröder et al., 2020). These imaging approaches, however, lack the temporal precision as in the electrophysiology, which is an important aspect of the information processing in the retina (Gollisch and Meister, 2008).

From our OT recordings, we examined the visual responses to a set of visual stimuli widely used for probing retinal function ex vivo (Baden et al., 2016; Jouty et al., 2018), including moving gratings, white-noise stimuli, and full-field flickering stimuli at different temporal frequencies. We used two different anesthetics that are commonly used in neuroscience research: isoflurane gas and an intraperitoneal combination of fentanyl, medetomidine and midazolam (FMM). In both cases, we found that the temporal dynamics of the retinal outputs were slower than in awake recordings, consistent with the previous studies on the effects of anesthesia in retinorecipient areas (dLGN, Durand et al., 2016; SC, De Franceschi and Solomon, 2018). We also found that the retinal outputs in an awake condition had higher baseline firing rates than in anesthetized or ex vivo conditions. As expected from previous ex vivo studies (Masland, 2012; Sanes and Masland, 2015; Baden et al., 2016; Jouty et al., 2018), anesthetized ON cells encoded light increments by increasing firing from their 
low baseline firing rates. In contrast, many awake ON cells encoded light decrements by decreasing firing from their high baseline firing rates, but not light increments due to saturation. They convey the same information in either condition: the more spikes, the higher light intensity. However, the amount of information transmitted per spike is lower in the awake responses. This disagrees with sparse efficient coding (Attneave, 1954; Barlow and Rosenblith, 1961) or minimum energy principles (Laughlin, 2001) that are widely used as a model of the early visual processing (Atick and Redlich, 1990; Gjorgjieva et al., 2019). A new

87 theoretical framework based on dense coding principles will thus be needed to explain retinal function in vivo.

\section{Results}

\section{Characterization of retinal output responses in vivo}

91 To monitor retinal output in vivo, we established extracellular single-unit recording methods

92 from axons of retinal ganglion cells (RGCs) in the optic tract (OT) of head-fixed mice (Figure

93 1). In total, we made 17 chronic (75 cells) and 52 acute recordings (298 cells with isoflurane;

94103 cells with FMM), where a standardized set of visual stimuli were presented to the subject animal to characterize the visual response properties of the recorded cells (for $\sim 1$ hour; Figure 1A,B; see Methods for details). 
A

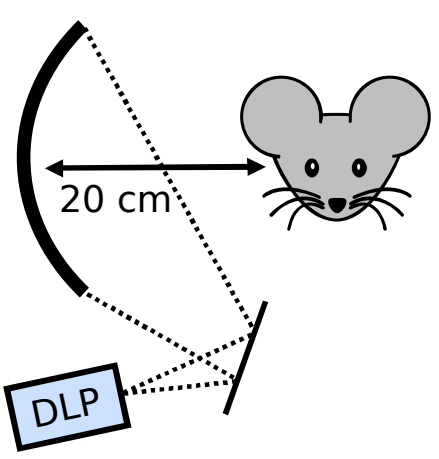

B

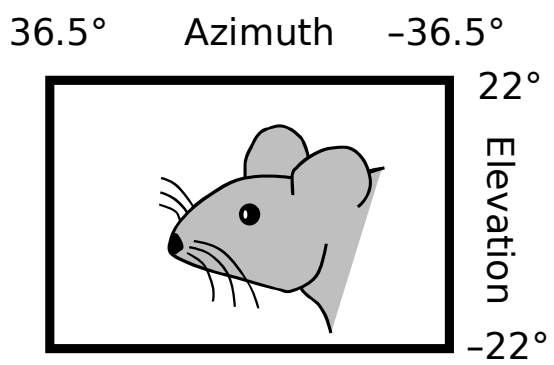

C

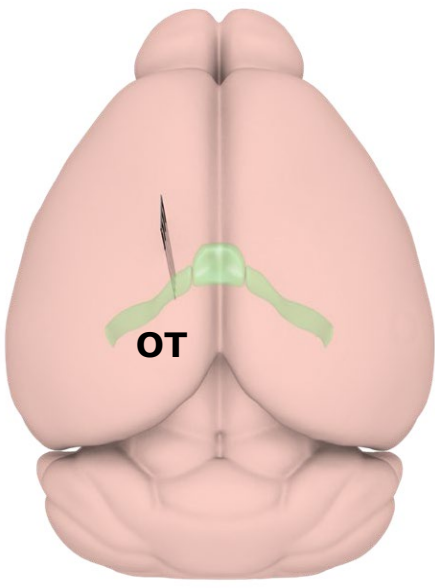

D

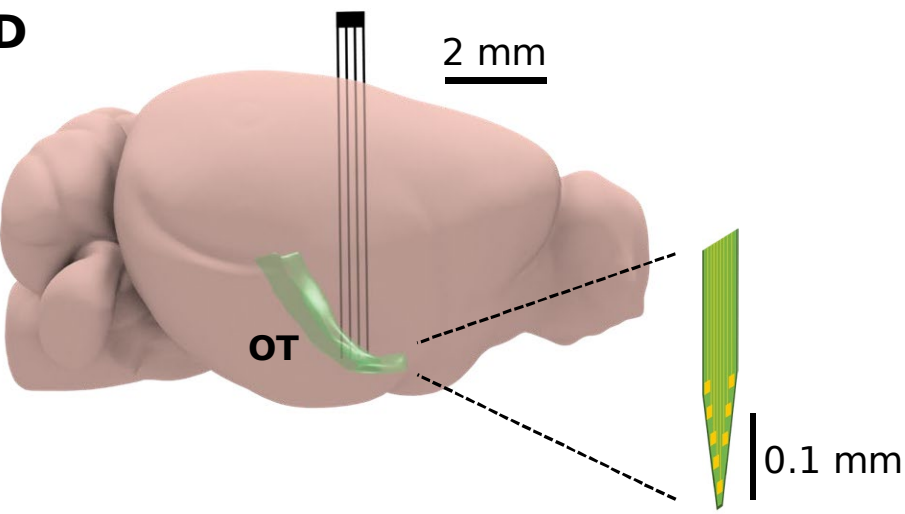

$\mathbf{E}$

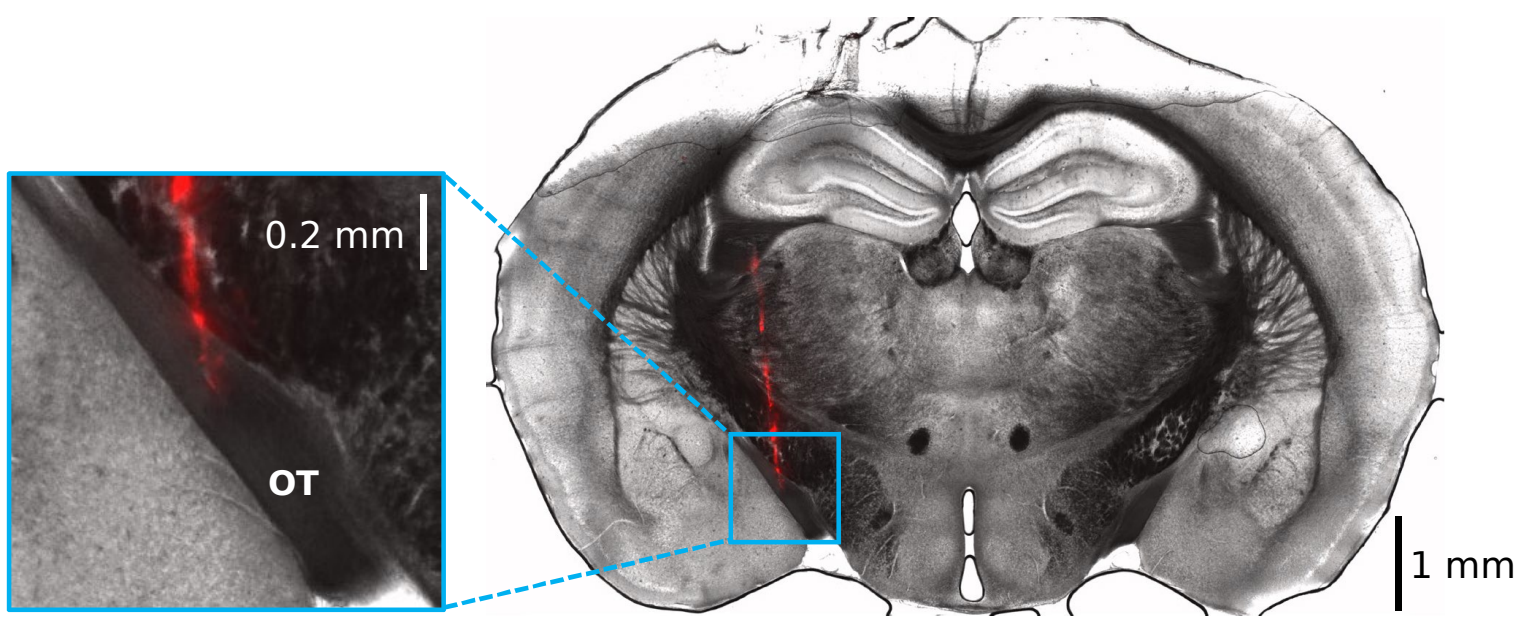

Figure 1: in vivo extracellular recordings from the mouse optic tract. $A, B$ : Schematic diagram of the experimental set-up. We presented visual stimuli to a head-fixed mouse using a digital light processing (DLP) device projecting images onto a spherical screen placed laterally to the subject animal (A, front-view; B, side-view). See Methods for details and specifications. $C, D$ : Schematic diagram ( $C$, top-view; $D$, side-view) of the brain and electrode location to target the optic tract (OT). E: Histological image of a representative brain sample (coronal section, $150 \mu \mathrm{m}$ thick) showing the electrode trace (red, Dil stain deposited on the electrode). 
We performed physiological cell-type classification of the recorded cells (see Methods

for details) and identified most major RGC types in our data sets (Figure 2; Sanes and Masland, 2015; Baden et al., 2016; Jouty et al., 2018). Specifically, we first identified reliably responsive cells based on the visual responses to full-field contrast-inverting stimuli (Figure 2A,B), and categorized their response polarity into ON, ON/OFF, OFF types (Figure 2C). In both anesthetized and awake conditions, we found around $30-50 \%$ of ON cells, $10-30 \%$ of ON/OFF cells, and around $20 \%$ of OFF cells in our data sets. We then further classified the cells from the viewpoint of motion sensitivity, based on the responses to moving gratings in eight different directions (Figure 2D-F). In the anesthetized conditions, about a half of the cells $(42-56 \%)$ were motion sensitive regardless of their response polarities or anesthetics. In contrast, we found much less motion sensitive cells (15\%) in the awake condition. This is consistent with the previous results in the superior colliculus (SC; Kasai and Isa, 2021), and presumably due to the consequence of optokinetic nystagmus (Kretschmer et al., 2017). When a motion in a wide visual field is presented, such as moving gratings, mice show compensatory eye movements to stabilize the image on the retina (Tabata et al., 2010). As a result, motion sensitive RGCs might have been driven less in the awake condition.

Our approach cannot obtain some RGC types that do not send their axons to OT, such as the one projecting to the suprachiasmatic nucleus via the retinohypothalamic tract ( $\mathrm{Li}$ and Schmidt, 2018). Nevertheless, the fact that we obtained diverse types of visual responses, better than previous in vivo studies (Hong et al., 2018; Liang et al., 2018; Schröder et al., 2020; Sibille et al., 2021) if not all the $~ 30$ RGC types observed ex vivo (Baden et al., 2016; Jouty et al., 2018), validates our recording methods to monitor retinal outputs in vivo. 

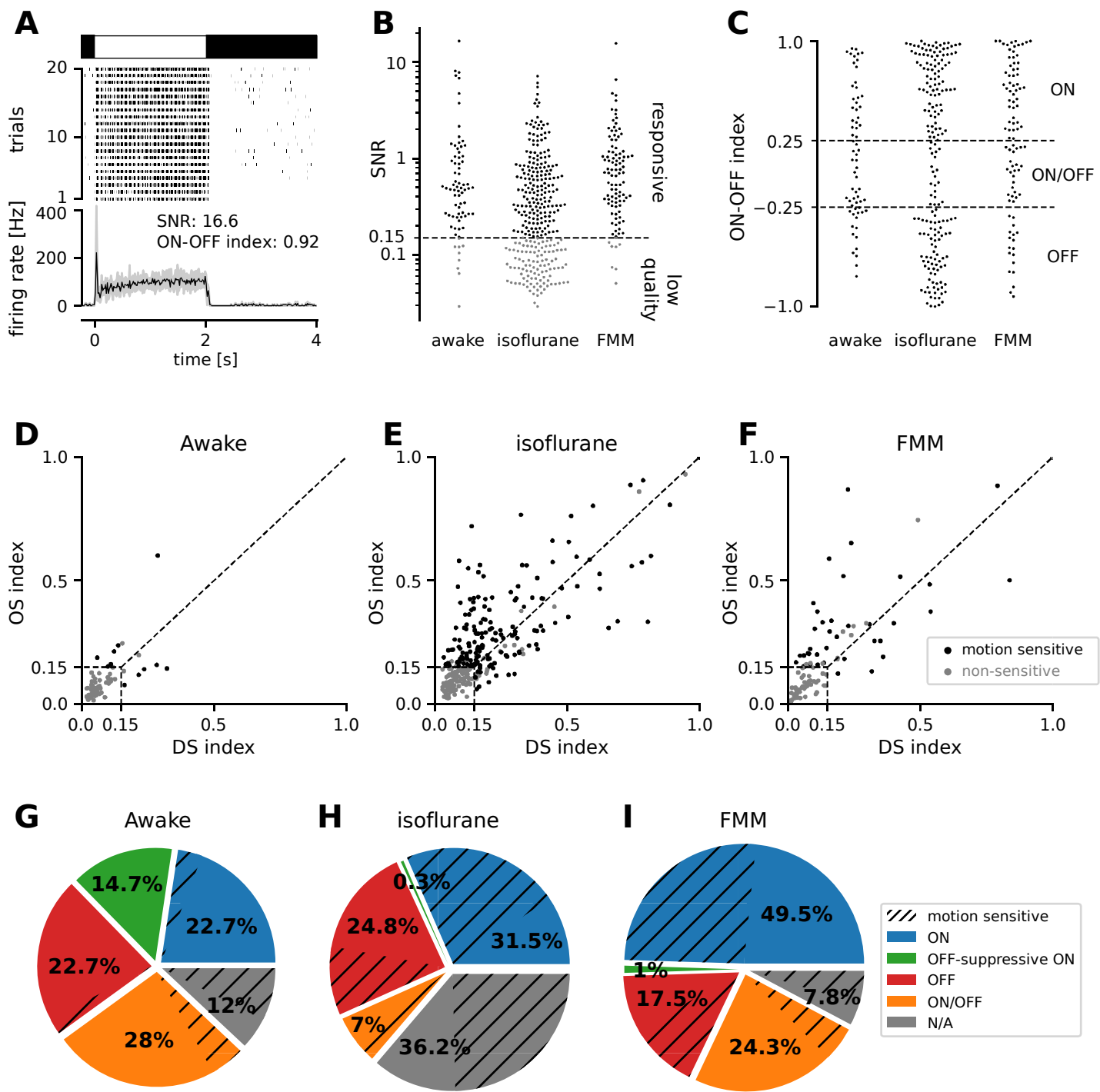

Figure 2: Physiological classification of retinal output responses in vivo. $A$ : Representative retinal output responses to full-field contrast inverting stimuli: top, stimulus; middle, raster graph over trials; bottom, peri-stimulus time histogram (black, mean; gray, variance; signal-to-noise ratio (SNR), Eq.(1) in Methods; ON-OFF index, Eq.(2)). B: SNR of the retinal output responses in different recording conditions. We set a threshold at 0.15 to identify reliably responsive cells (black) and low-quality unclassifiable cells (gray). C: ON-OFF index distributions from the reliably responsive cells. While no apparent clusters were identified, we set a threshold at \pm 0.25 to categorize the response polarity into ON, ON/OFF, and OFF types. Within the ON cell type, we further identified an OFF-suppressive type based on the full-field flickering stimulus responses (Figure 3). D-F: Distribution of DS/OS indices (Eq.(3)) in each recording condition (D, awake; $E$, isoflurane; F, FMM). We set a threshold at 0.15 (with $p<0.2$ ) to identify motion sensitive (black) and non-sensitive (gray) cells. G-l: Fraction of identified cell types in vivo: ON (blue; OFF-suppressive type in green), OFF (red), ON/OFF (orange), and the rest unclassifiable cells ("N/A", gray). Cells in each category were further divided based on the motion selectivity (hatched). The OFF-suppressive ON cells were prominent in the awake condition $(G, 11 / 75$ cells), but rarely observed under anesthesia $(H$, isoflurane, 1/298 cells; I, FMM, 1/103 cells). 


\section{Higher firing in awake than in anesthetized mice}

147 For characterizing the retinal output properties in vivo, we first analyzed the responses to full-

field sinusoidally flickering stimuli that linearly changed the amplitude over time (from 0 to $100 \%$ contrast for $10 \mathrm{~s}$; see Figure 3A-C for representative responses). An even power of sine function with a sigmoid envelope generally fitted well to the responses (Eq. (6) in Methods). We then focused on the parameter values to analyze the response properties in different recording conditions.

First, we found significantly higher baseline firing rates in awake mice $(18 \pm 26 \mathrm{~Hz}$; median \pm interquartile range) than in those under anesthesia (isoflurane, $2 \pm 6 \mathrm{~Hz}, p<0.001$, U-test; FMM, $2 \pm 4 \mathrm{~Hz}, p<0.001$; Figure 3D). Awake ON cells had particularly high baseline activity (up to $\sim 100 \mathrm{~Hz}$ ) and showed a prominent reduction in firing rates in response to light decrements (see Figure 3B for example). In contrast, due to this high baseline activity near saturation, many of them showed virtually no responses to light increments $(N=11 / 28)$, except for a sharp rebound response to a full-contrast inversion $(N=8 / 11$; see Figures $2 \mathrm{~A}$ and 3B for example). These ON cells had negative amplitudes in the curve fit (Figure 3E, green) with the same response phase as OFF cells (Figure $3 \mathrm{~F}$; see Figure $3 \mathrm{~A}$ for a representative OFF cell's responses). This indicates that they primarily encode light decrements by decreasing firing from their high baseline firing rates $(61 \pm 27 \mathrm{~Hz}$, mean \pm standard deviation), rather than light increments by increasing firing as conventional ON cells do (Masland, 2012; Sanes and Masland, 2015; Baden et al., 2016; Jouty et al., 2018). We thus categorized these cells independently as an "OFF-suppressive" ON type (Figure 2G). This is a new response type likely emerged due to high baseline firing in awake mice. Indeed, such responses were barely observed in anesthetized animals (isoflurane, 1/298 cells; FMM, $1 / 103$ cells; Figure $2 \mathrm{H}, \mathrm{I}$ ) where the baseline firing rate was generally low (Figure 3D). As expected from previous ex vivo studies (Masland, 2012; Sanes and Masland, 2015; Baden et al., 2016; Jouty et al., 2018), anesthetized ON cells increased firing upon light increments (see 
173

an opposite response phase from OFF cells (Figure 3F) further support that these ON cells encode light increments. Taken together, these data suggest that the retinal coding depends on the baseline firing level of RGCs.

To characterize contrast sensitivity, we next examined the estimated response magnitude at $10 \%$ contrast from the sigmoid function used in the curve fit (Eq.(6)), and found no significant differences across the recording conditions (awake, $4 \pm 10 \mathrm{~Hz}$, median \pm interquartile range; isoflurane, $6 \pm 13 \mathrm{~Hz}$; FMM, $3 \pm 10 \mathrm{~Hz} ; p=0.13$, Kruskal-Wallis test). Nevertheless, the midpoint of the sigmoid was larger in awake cells $(67 \pm 43 \%$ contrast, median \pm interquartile range) than in anesthetized ones (isoflurane, $45 \pm 44 \%, p<0.001$, Mann-Whitney U-test; FMM, $43 \pm 38 \%, p=0.002$ ) so was the plateau (absolute value of peak evoked firing rate, Figure $3 \mathrm{E}$; awake, $52 \pm 68 \mathrm{~Hz}$, median \pm interquartile range; isoflurane, $37 \pm 33 \mathrm{~Hz}, p=0.03$, Mann-Whitney U-test against awake data; FMM, $35 \pm 34 \mathrm{~Hz} ; p=$ 0.007). This indicates a larger dynamic range in the awake condition, while contrast sensitivity itself remains largely unchanged.

Finally, we found a significant phase shift in the responses between the recording conditions (Figure 3F). This phase parameter in the curve fit represents the position of the response peak relative to the sinusoidal stimulus intensity patterns (see Methods for details), and we identified two clusters in each data set: the one with negative phase for ON cells (Figure 3F, blue) and the other with positive phase for OFF and OFF-suppressive ON cells (Figure 3F, red and green, respectively). ON/OFF cells were found in either cluster, depending on the relative strength of their responses to light increments versus light decrements (Figure $3 F$, orange). In both clusters, we found that the phase was on average smallest for the awake condition ( $-2.4 \pm 0.3$ and $1.5 \pm 0.3$ radian, respectively; mean \pm standard deviation; isoflurane, $-0.8 \pm 0.5$ and $2.0 \pm 0.5$ radian; $p<0.001$ for both cases, $t$-test), while largest for the FMM anesthesia $(-0.2 \pm 0.8$ and $2.6 \pm 0.6$ radian; $p<0.001$ for both cases against corresponding isoflurane data). This implies that the response dynamics are faster in awake mice than those under anesthesia, especially with FMM. 


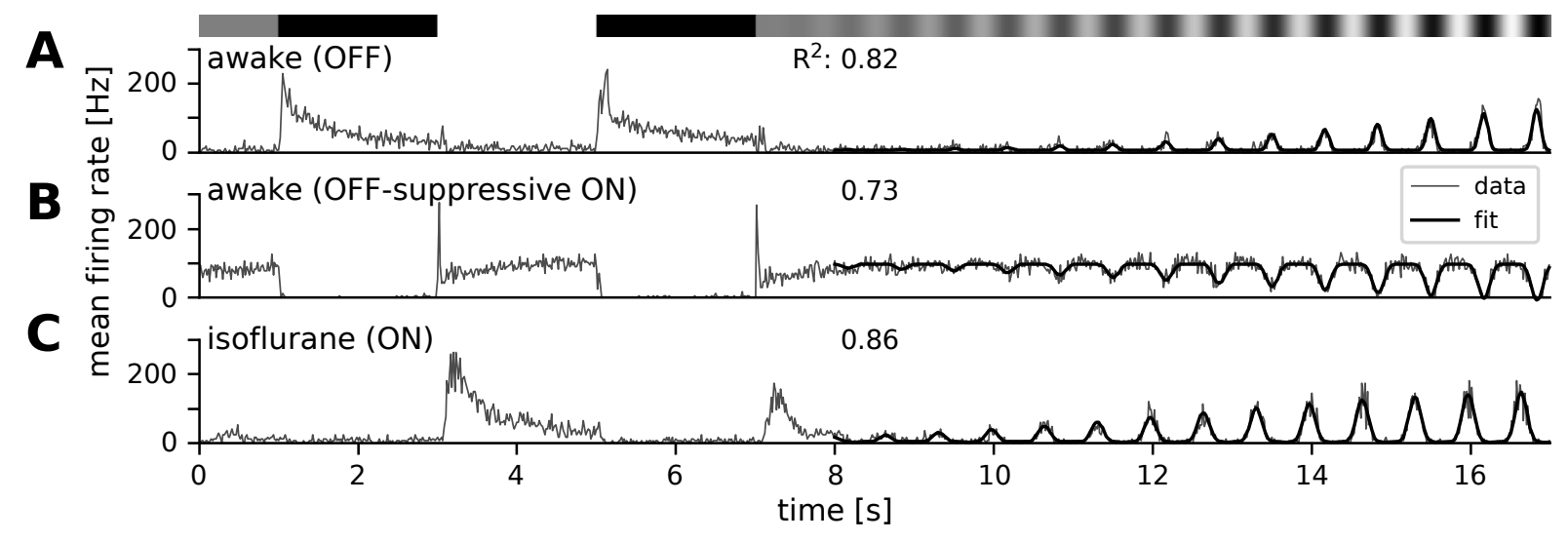

D

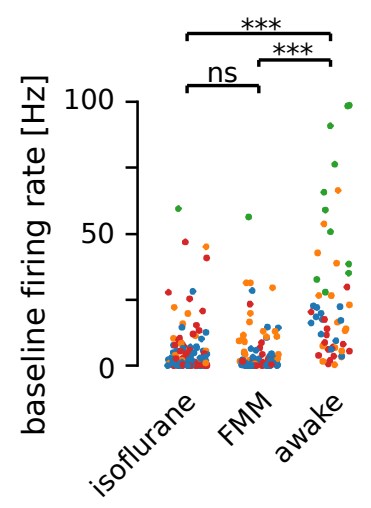

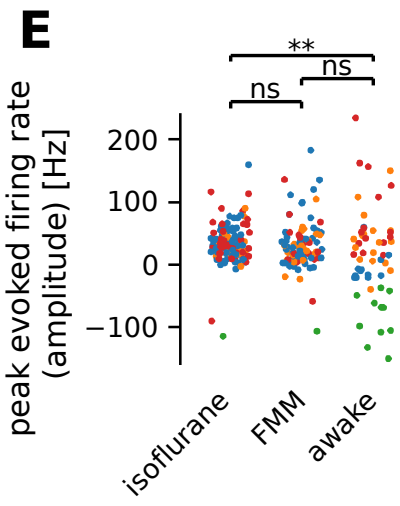

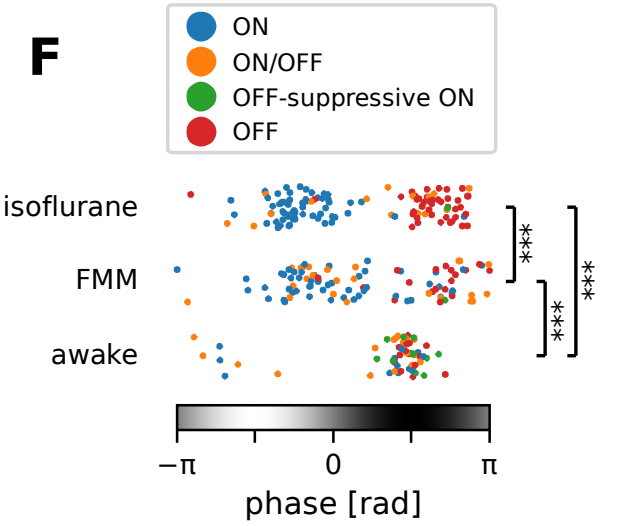

Figure 3: Many ON retinal ganglion cells showed suppressive OFF responses in awake condition. A-C: Mean firing rate of representative cells in response to a sinusoidally-flickering stimulus with increasing contrast in the awake (A, OFF; B, OFF-suppressive ON, the same cell as in Figure 2A) or anesthetized conditions ( $C, O N)$. Overlaid with the peri-stimulus time histogram (gray) is the model fit (black, Eq.(6) in Methods). The number on top indicates the fit quality (explained variance $R^{2}$ in Eq.(5)). D-F: population data of the model parameters (D, baseline $B$; $\mathrm{E}$, amplitude $A ; \mathrm{F}$, phase $\phi$ ) across different conditions: isoflurane anesthesia $(N=103)$, FMM anesthesia $(N=92)$ and awake $(N=51)$. The sinusoidal stimulus pattern relative to the response peak is also indicated at the bottom of $\mathrm{F}$. Cell types are color-coded as in Figure 2 (blue, ON; green, OFF-suppressive ON; red, OFF; orange, ON/OFF). Note high baseline with negative amplitude and positive phase for the OFF-suppressive ON cells, which were predominantly found in the awake condition: ${ }^{* * *}, p<0.001$; ${ }^{* *}, p<0.01$; ns, nonsignificant (D, U-test; $E, F$-test; $F, t$-test). 


\section{Faster response kinetics in awake than in anesthetized mice}

215 To better characterize the kinetics of retinal output in vivo, we next analyzed the responses to

216 full-field sinusoidal flickers at different temporal frequencies (Figure 4). In awake mice, most

217 cells responded faithfully to all the stimulation frequencies we tested from 1.875 to $15 \mathrm{~Hz}$ (see

218 Figure $4 \mathrm{~A}$ for example). Some awake cells showed the largest responses to the $15 \mathrm{~Hz}$

219 stimulation (Figure 4A; curve fit in violet, Eq. (4)), suggesting that they were possibly tuned to

220 even higher frequencies. In the anesthetized conditions, in contrast, retinal output responses

221 typically followed the stimulation frequencies up to $7.5 \mathrm{~Hz}$, but failed to do so at $15 \mathrm{~Hz}$ (see

222 Figure 4B for example). For quantification, we fitted an even power of sine function to the

223 responses (Eq.(4) in Methods) and set a threshold of the curve fit quality (defined as the

224 explained variance; Eq.(5)) at 0.2 to select cells with robust responses (Figure 4C,D). In the

225 awake condition, a larger number of cells responded well at medium frequencies $(3.75 \mathrm{~Hz}$,

$22675 \% ; 7.5 \mathrm{~Hz}, 82 \%)$ than at a low frequency $(1.875 \mathrm{~Hz}, 60 \%)$; and the majority of the awake cells remained responsive to a high frequency stimulus $(15 \mathrm{~Hz}, 58 \%)$. Under anesthesia, in contrast, the fraction of responsive cells was the largest at a low frequency $(1.875 \mathrm{~Hz}$; isoflurane, $85 \%$; FMM, 67\%). Fewer cells responded robustly at faster flicker rates $(3.75 \mathrm{~Hz}$ and $7.5 \mathrm{~Hz}$; isoflurane, $73 \%$ and $65 \%$, respectively; $\mathrm{FMM}, 63 \%$ and $59 \%$ ), and only a small fraction of the anesthetized cells were able to follow the stimulation frequency at $15 \mathrm{~Hz}$ (isoflurane, 9\%; FMM, 3\%). Consistent with the phase analysis results described above (Figure 3F), these outcomes indicate that the retinal output dynamics are faster in awake animals than those under anesthesia, especially for FMM. 


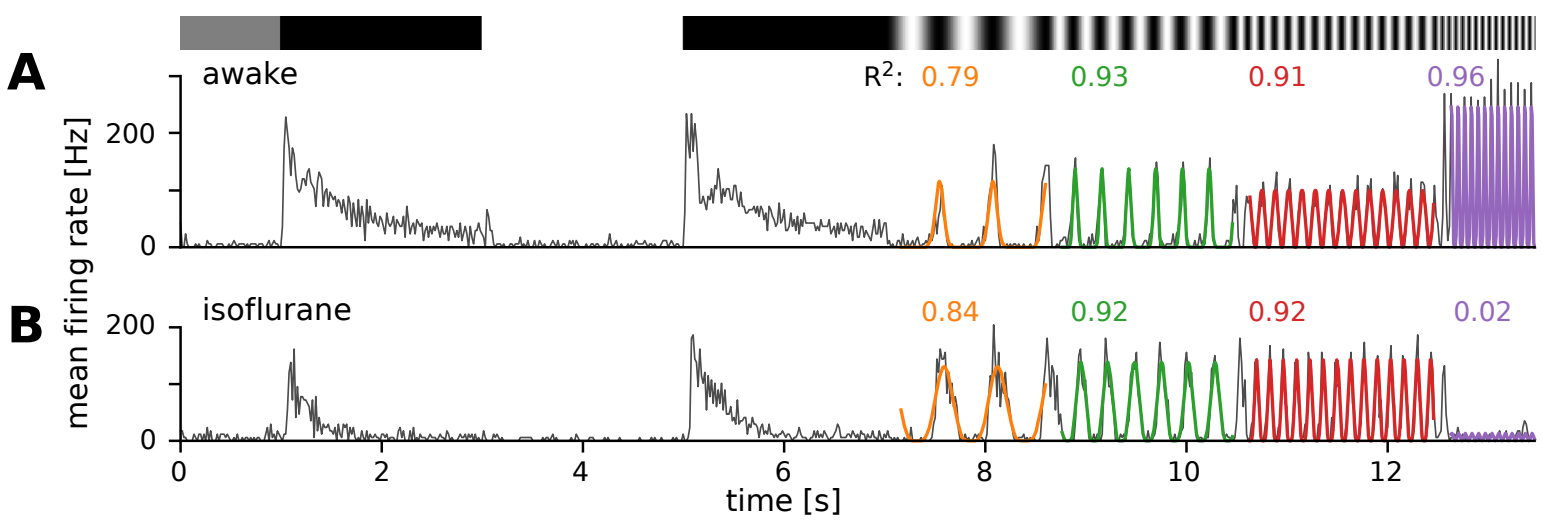

C

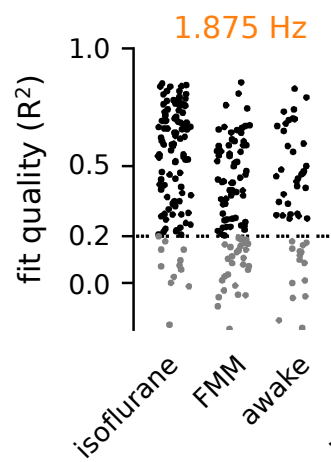

$3.75 \mathrm{~Hz}$

$7.5 \mathrm{~Hz}$

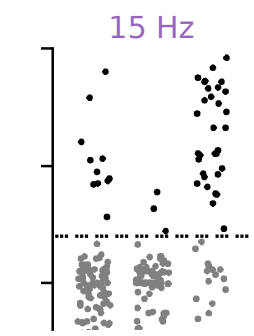

\section{D}

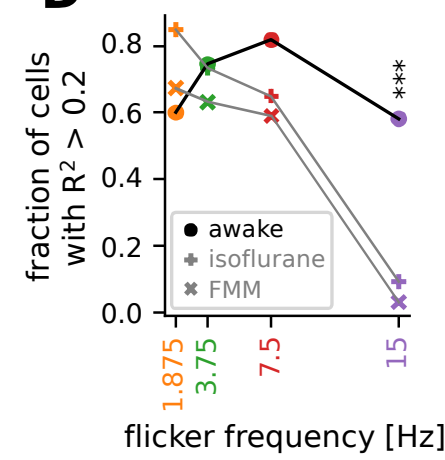

Figure 4: Retinal output showed higher temporal frequency sensitivity in awake than in anesthetized mice. A: Representative retinal output (black, mean firing rate over 10 trials) in an awake condition in response to full-field sinusoidally flickering stimuli at different temporal frequencies $(1.875,3.75,7.5$ and $15 \mathrm{~Hz})$, following full-field contrast inversions. Overlaid is the curve fit (Eq.(4) in Methods) in different colors. The number on top is the explained variance of the curve fit ( $R^{2}$, Eq. (5) in Methods) in corresponding colors, representing the fit quality. $B$ : Representative retinal output responses under isoflurane anesthesia (shown in the same format as in A). C: Population data of the fit quality at four different stimulus frequencies in the awake $(N=51)$ or anesthetized conditions (isoflurane, $N=103$; FMM, $N=92$ ). The fit quality threshold was set to be 0.2 (black, $R^{2} \geq 0.2$; gray, $R^{2}<0.2$ ). $D$ : Fraction of the cells with the fit quality above the threshold across different conditions (awake, black line with circles; isoflurane, gray line with vertical crosses; FMM, gray line with diagonal crosses), representing the frequency tuning of the retinal output at the population level. A significantly larger fraction of cells was responsive at $15 \mathrm{~Hz}$ in the awake condition than in the anesthetized conditions $\left({ }^{* * *}, p<0.001\right.$ for both isoflurane and FMM; two-proportion $z$-test). 


\section{Comparison of retinal output properties between in vivo}

\section{and ex vivo}

253

254

255

256

We have thus far focused on the retinal output properties in vivo, and showed higher baseline firing rates (Figure 3) and faster response kinetics (Figure 4) in awake mice than in those under anesthesia. We next asked if RGC responses differ between in vivo and ex vivo conditions. This is a critical comparison because retinal physiology has been mostly studied and best characterized in an isolated preparation (Gollisch and Meister, 2010; Sanes and Masland, 2015), but little is known about it in awake animals (Weyand, 2007; Liang et al., 2018, 2020; Schröder et al., 2020; Sibille et al., 2021). Here we exploited stimulus ensemble statistical techniques ("reverse correlation"; Meister et al., 1994; Chichilnisky, 2001) to systematically characterize the visual response properties and make a direct comparison across different recording conditions in the linear-nonlinear cascade model framework (see Methods for details). Specifically, using full-field white-noise stimuli, we analyzed 1) the linear temporal filter (Figure 5), estimated by the spike-triggered average (STA) stimulus, i.e., the mean stimulus that triggered spiking responses; and 2) the static nonlinear gain function (Figure 6), i.e., an instantaneous mapping of the STA output to the neural responses (Eq. (7) in Methods). Here we reanalyzed the existing data sets for ex vivo recordings (696 cells from 18 isolated mouse retinas; Vlasiuk and Asari, 2021).

\section{Faster response kinetics in awake condition than ex vivo}

We identified a good quality STA in more than two-thirds of the cells recorded in vivo (e.g., Figure 5A). To compare the temporal dynamics of the STAs across different recording conditions, we used the following two measures: 1) the first peak latency of the STA estimated from a difference-of-Gaussian curve fit (Figure 5A); and 2) spectral peak frequency calculated by the Fourier transform of the fitted curve (Figure 5B). Consistent with the results measured by the flickering stimuli at different frequencies (Figure 4), we found faster kinetics in awake animals than in anesthetized ones (Figure 5C,D): i.e., significantly shorter peak latencies 
277

278

279

280

281

282

283

284

285

286

287

288

289

290

291

292

293

294

295

296

297

(awake, $35 \pm 12 \mathrm{~ms}$, mean \pm standard deviation; isoflurane, $79 \pm 18 \mathrm{~ms}, p<0.001$, $t$-test; FMM, $87 \pm 24 \mathrm{~ms}, p<0.001$; Figure 5C) and higher peak frequencies (awake, $9.6 \pm 3.0 \mathrm{~Hz}$; isoflurane, $4.6 \pm 1.6 \mathrm{~Hz}, p<0.001$; FMM, $3.7 \pm 1.2 \mathrm{~Hz}, p<0.001$; Figure 5D). Interestingly, we identified that the ex vivo data lied in-between. The ex vivo STAs had significantly longer peak latencies $(64 \pm 28 \mathrm{~ms}, p<0.001)$ and lower peak frequencies $(4.3 \pm 1.8 \mathrm{~Hz}, p<0.001)$ than the awake ones. In contrast, the peak latencies were significantly shorter in an isolated retinal preparation than in the anesthetized conditions (isoflurane, $p<0.001 ; \mathrm{FMM}, p<0.001$ ), while the peak frequencies were comparable between these conditions (isoflurane, $p=0.7$; FMM, $p=0.052)$.

We further analyzed the STA dynamics across different cell types. For clustering the STAs obtained in each recording condition, we used the principal component analysis (PCA; see Methods for details). As in previous ex vivo studies (Gollisch and Meister, 2008; Asari and Meister, 2014), the first two principal components accounted for most of the variance $(78-86 \%$, collectively), and the four quadrants of the PCA biplot generally represented distinct filter shapes, corresponding to monophasic OFF (mono-OFF), biphasic OFF (bi-OFF), monophasic ON (mono-ON), and biphasic ON (bi-ON) response types, respectively. In all recording conditions, no apparent cluster was found in this feature space, leading to a continuum of the STA shape patterns across populations (Figure 5E-H). Nevertheless, we identified two features that were different between ex vivo and in vivo, especially distinct in the awake condition. First, while the monophasic types were generally slower than the biphasic types, differences in the peak latencies were much larger in the ex vivo (Figure 5G; mono-ON $83 \pm$ 24 ms versus bi-ON $58 \pm 31 \mathrm{~ms}, p<0.001$, $t$-test; mono-OFF $67 \pm 29$ ms versus bi-OFF $48 \pm$ $8 \mathrm{~ms}, p<0.001$ ) or anesthetized conditions (Figure 5E, isoflurane: mono-ON $91 \pm 22 \mathrm{~ms}$, biON $67 \pm 6 \mathrm{~ms}, p<0.001$; mono-OFF $89 \pm 14 \mathrm{~ms}$, bi-OFF $68 \pm 8 \mathrm{~ms}, p<0.001$; Figure $5 \mathrm{~F}$, FMM: mono-ON $97 \pm 30 \mathrm{~ms}$, bi-ON $78 \pm 11 \mathrm{~ms}, p=0.051$; mono-OFF $103 \pm 13 \mathrm{~ms}$, bi-OFF $70 \pm 11 \mathrm{~ms}, p<0.001$ ) than in the awake condition (Figure $5 \mathrm{H}$; mono-ON $40 \pm 7 \mathrm{~ms}$, bi-ON $30 \pm 13 \mathrm{~ms}, p=0.03$; mon-OFF $39 \pm 7 \mathrm{~ms}$, bi-OFF $34 \pm 14 \mathrm{~ms}, p=0.2$ ). Second, we found 
bioRxiv preprint doi: https://doi.org/10.1101/2022.02.15.480512; this version posted February 17, 2022. The copyright holder for this preprint (which was not certified by peer review) is the author/funder. All rights reserved. No reuse allowed without permission.

Boissonnet, Tripodi, and Asari

304 that ON cells were significantly slower than OFF cells ex vivo (mono-ON versus mono-OFF,

$305 p=0.002$; bi-ON versus bi-OFF, $p=0.03$ ), but not in vivo $(p>0.2$ in all the conditions

306 examined). Taken together, our results suggest that awake responses are faster and their

307 kinetics are more similar across different cell types than ex vivo or anesthetized responses.
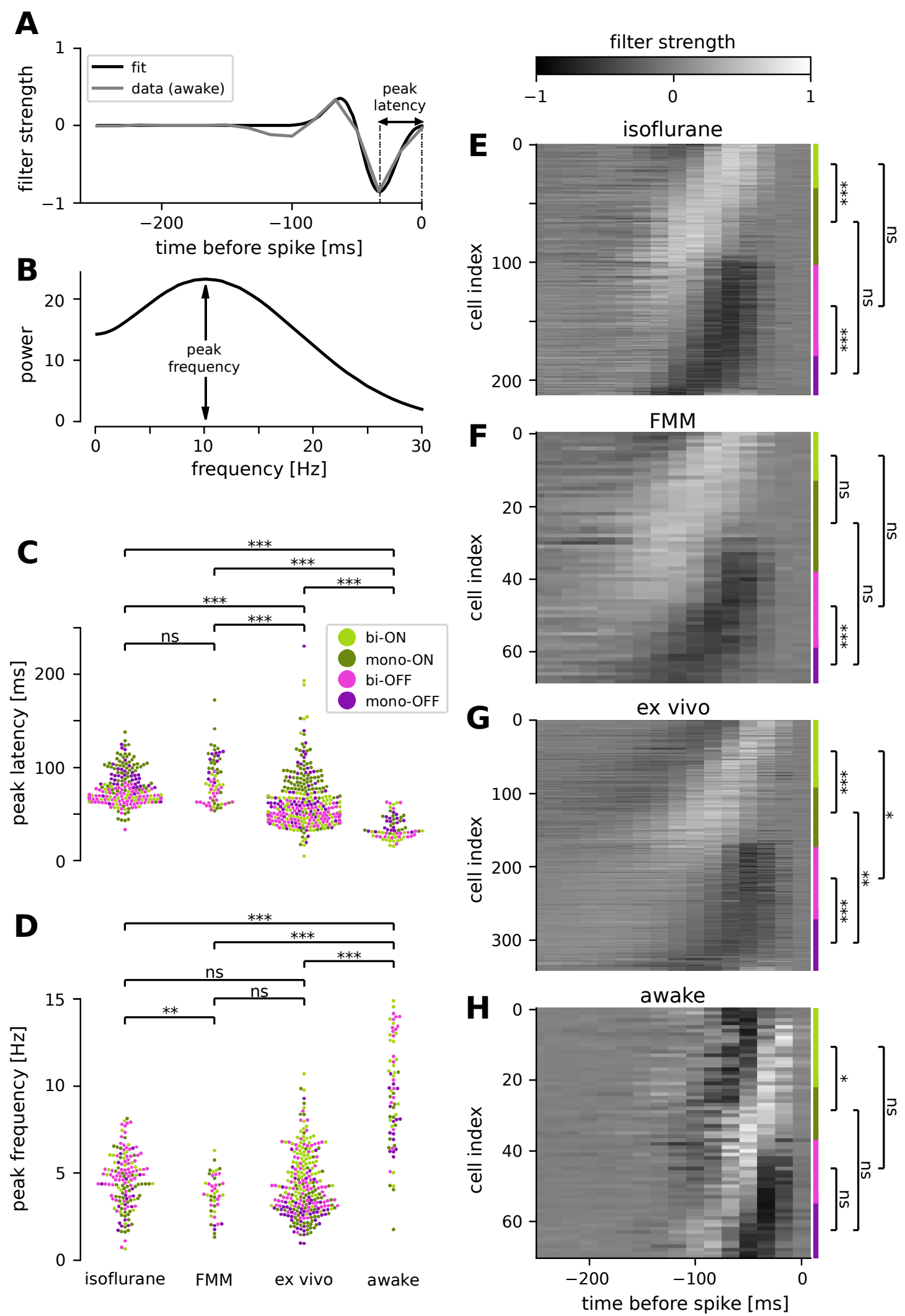
Figure 5: Retinal ganglion cells showed faster response dynamics in awake condition than in anesthetized or ex vivo conditions. A: Temporal filter of a representative awake cell (gray, spike-triggered average (STA) of the full-field randomly flickering stimulus) and a difference-of-Gaussian curve fit (black) for estimating the latency of the first peak. B: Power spectra of the example filter in A, based on the curve fit, for estimating the peak frequency. $C, D$ : Population data of the peak latencies (C) and frequencies (D) across different conditions (light green, biphasic ON; dark green, monophasic ON; pink, biphasic OFF; violet, monophasic OFF). Here and thereafter, ${ }^{* * *}, p<0.001 ;{ }^{* *}, p<0.01 ;{ }^{*}, p<0.05$; ns, non-significant (t-test). The filter types were identified by the quadrants of the PCA biplot (see Methods for details). $E-H$ : Population data of the temporal filters across different conditions: from top to bottom, isoflurane anesthesia (E, $p<N=213)$, FMM anesthesia ( $\mathrm{F}, N=69)$, ex vivo $(\mathrm{G}, N=342)$ and awake $(\mathrm{H}, N=71)$. The four filter types are indicated on the right with corresponding colors.

\section{Higher spiking activity in awake condition than ex vivo}

How does a recording condition affect the firing properties of RGCs? The mean firing rate during the white-noise stimulus presentation was significantly higher in awake $(35.0 \pm 27.3 \mathrm{~Hz}$; mean \pm standard deviation) than in all the other recording conditions (isoflurane, $11.0 \pm 10.2$ $\mathrm{Hz}$; FMM, $10.9 \pm 10.7 \mathrm{~Hz}$; ex vivo, $6.9 \pm 6.6 \mathrm{~Hz}$; all with $p<0.001, t$-test in the logarithmic scale; Figure 6A). Importantly, this is not due to differences in the stimulus condition because the light intensity level was overall comparable between the recording setups (in vivo, $\sim 16$ $\mathrm{mW} / \mathrm{m}^{2}$ on average at the eye; ex vivo, $\sim 18 \mathrm{~mW} / \mathrm{m}^{2}$ on the isolated retina; see Methods for details).

For a further analysis, we examined the static nonlinear gain function of the recorded cells (Eq. (7) in Methods). This gain function accounts for nonlinearity associated with spike generation, such as spike threshold and firing rate saturation, and is generally well explained by a sigmoid function (see Figure 6B for example). For quantification, we assessed the neutral firing rate of the cells where the input to the gain function is zero, i.e., the firing rate in the 
on the baseline firing rates measured by the sinusoidally flickering stimuli (Figure 3 ), the neutral firing rate was significantly higher in the awake condition $(29.5 \pm 25.4 \mathrm{~Hz}$, mean \pm standard deviation) than those under anesthesia (isoflurane, $7.6 \pm 8.4 \mathrm{~Hz}, p<0.001$, $t$-test on a log scale; FMM, $7.5 \pm 9.3 \mathrm{~Hz}, p<0.001$; Figure $6 \mathrm{C}$ ). We also found that the neutral firing rates ex vivo $(5.5 \pm 7.1 \mathrm{~Hz})$ were as low as those in the anesthetized conditions (isoflurane, $p=0.02 ; \mathrm{FMM}, p=0.1)$, and significantly lower than those in the awake condition $(p<0.001)$.

We further identified two distinct features in the gain function properties between ex vivo and in vivo responses. First, ON cell types generally had a higher gain than OFF cell types ex vivo (Figure 6F), whereas such cell-type specific differences were not observed in vivo (isoflurane, Figure 6D; FMM, Figure 6E; awake, Figure 6G). Second, the midpoint of the sigmoid fitted to ex vivo responses $(1.6 \pm 1.4$, median \pm interquartile range) was significantly higher than that for in vivo responses (isoflurane, $1.1 \pm 0.9$; FMM, $1.1 \pm 0.8$; awake, $0.9 \pm 1.2$; all with $p<0.001$, Mann-Whitney $U$-test; Figure $6 \mathrm{H}$ ). Taken together with the outcomes obtained with the sinusoidally flickering stimulus (Figure 3 ), this suggests that in vivo responses are more linearized to cover a larger dynamic range, especially in the awake condition due to high neutral responses (Figure 6G). 

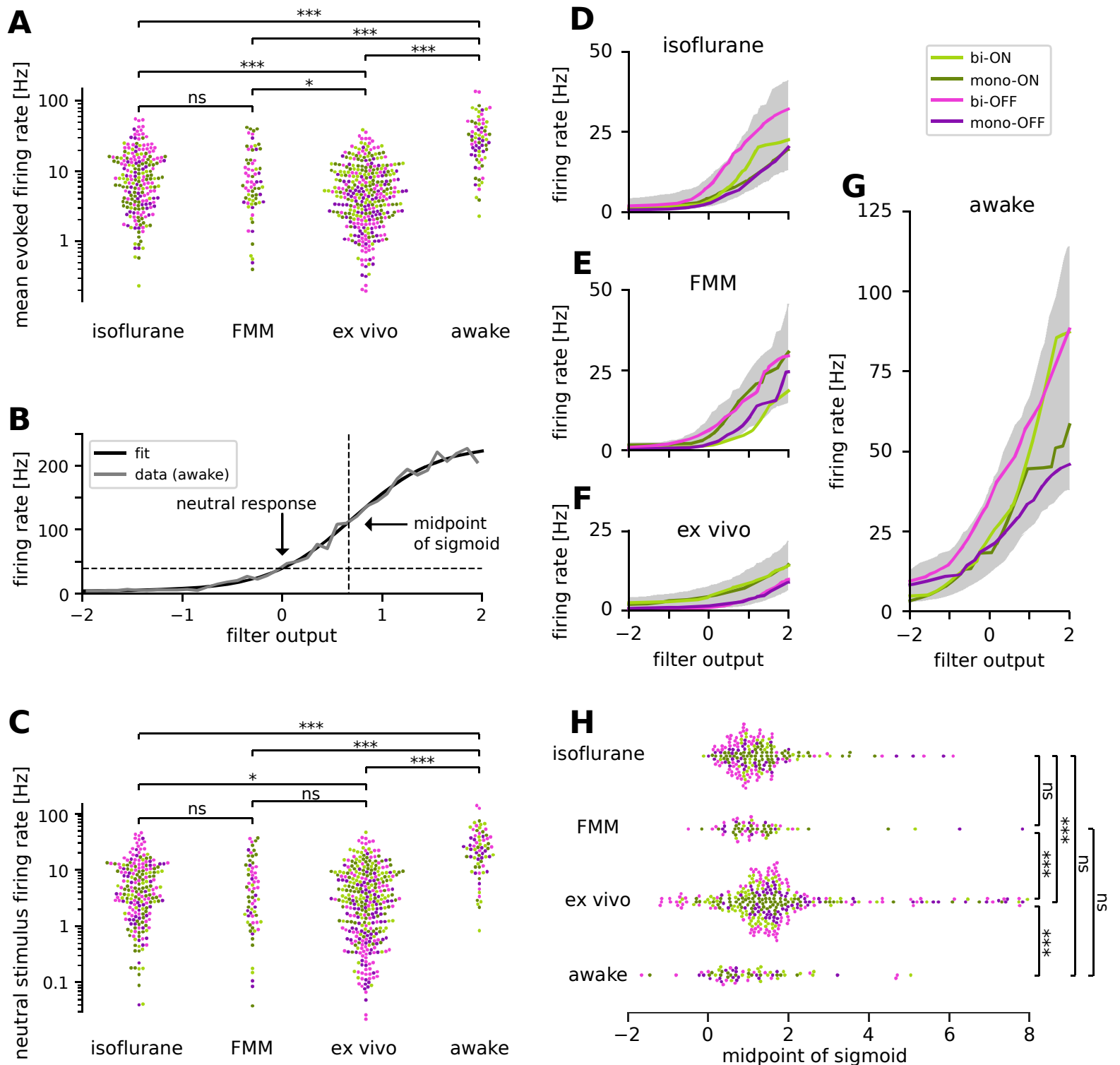

Figure 6: Retinal ganglion cells showed higher firing activity in awake condition than in anesthetized or ex vivo conditions. Cell types are color-coded as in Figure 5 (light green, biphasic ON; dark green, monophasic ON; pink, biphasic OFF; violet, monophasic OFF). $A$ : Population data of the mean firing rates during the stimulus presentation period in four different recording conditions (isoflurane, FMM, ex vivo, and awake): ${ }^{* *}, p<0.001 ;{ }^{* *}, p<0.01 ;{ }^{*}, p<$ 0.05 ; ns, non-significant ( $t$-test on the logarithm of firing rates). $B$ : Static nonlinear gain function of a representative awake cell (the same one as in Figure $5 \mathrm{~A}$ ), estimated by the stimulus ensemble statistical techniques applied to the responses to a full-field randomly flickering stimulus (gray, Eq.(7) in Methods; black, sigmoid curve fit with the midpoint at 0.71). Note a high neutral stimulus response $(40 \mathrm{~Hz})$ defined as the firing rate at zero filter output (i.e., in the presence of stimuli orthogonal to the cell's STA). C: Population data of the neutral stimulus responses in each recording condition (in the same format as in A). D-G: Population data of the static nonlinear gain function (median for each cell type in corresponding colors; gray, interquartile range of all cells) across different conditions: isoflurane anesthesia ( $D, N=213)$, FMM anesthesia $(\mathrm{E}, N=69)$, ex vivo $(\mathrm{F}, N=342)$, and awake condition $(\mathrm{G}, N=71)$. $H$ : Population data of the midpoint of the sigmoid nonlinearity in each recording condition: ${ }^{* * *}$, $p<0.001$; Mann-Whitney $U$-test. 


\section{1}

372

373

\section{Discussion}

Here we established optic tract recordings in head-fixed mice (Figure 1) and systematically analyzed the retinal output properties in vivo using a standardized set of visual stimuli for characterizing retinal functions (Baden et al., 2016; Jouty et al., 2018). We found that awake response properties were overall similar to those under isoflurane or FMM anesthesia (Figures 2-4) or ex vivo (Figures 5 and 6); however, we identified two distinct features in the awake condition: higher firing rates (Figures 3 and 6) and faster response dynamics (Figures 4 and 5). The change in the kinetics likely arose as a direct consequence of the high baseline activity in awake animals. When cells are on average more depolarized, it is faster to reach the spike threshold upon stimulation, hence leading to a shorter response latency (Zohar et al., 2011). High firing activity in awake animals has been widely observed in many brain areas across species (Greenberg et al., 2008; Sellers et al., 2015; Durand et al., 2016; Wright et al., 2017; De Franceschi and Solomon, 2018; Chen and Song, 2019). Therefore, the retinal response characteristics we observed in vivo could be a general feature of the brain in an awake state.

In awake animals, both the baseline and visually-evoked firing rates of RGCs were much higher —on average by $\sim 20 \mathrm{~Hz}$-than in those under anesthesia or in an isolated preparation (Figures 3 and 6). As a result, many awake ON cells showed OFF-suppressive responses, a new response type observed only in those cells with high baseline firing rates $(\sim 60 \mathrm{~Hz})$. Here we classified these cells separately from the other ON cells in our clustering analysis (Figure 2); however, it is unlikely that they form a distinct "cell type" in the retina. Instead, these cells likely belong to a known cell type, and altered their response properties due to an increased baseline activity in the awake condition. Possible candidate cell types are: transient $\mathrm{ON}$ alpha cells (although they have a low baseline firing rate ex vivo; Krieger et al., 2017), ON contrast suppressive cells (although a sharp rebound response is missing in the calcium dynamics ex vivo; Baden et al., 2016), or suppressed-by-contrast cells (although their high baseline activity is suppressed by both ON and OFF stimuli; Mastronarde, 1985; Tien et 
al., 2015; Jacoby and Schwartz, 2018). It is interesting to identify the exact cell type of these RGCs and further characterize their function in vivo in future studies.

What are the mechanisms underlying such differences between in vivo and ex vivo retinal responses? One possibility is the difference in the physiological states of the retina. Even if ex vivo recordings are made under a proper temperature using a perfusion medium specifically formulated to support isolated retinal tissues (Ames and Nesbett, 1981), there are certain unavoidable differences to the physiological condition in vivo. In particular, the retinal pigment epithelium, a key player for the visual cycle and spatial buffering of ions, is often removed in an isolated retinal preparation. A lack of retinal supply can then alter the physiological states of the retinal neurons, potentially leading to a change in their visual response properties.

Differences in the input stimulus properties, in contrast, cannot explain our results, even though retinal responses depend a lot on stimulus conditions. For example, the higher the light intensity level is, the higher the temporal frequency sensitivity becomes for ex vivo RGC responses (threshold at around 20-30 Hz; Wang et al., 2011) and so does the critical flicker-fusion frequency at the behavioral level $(15-40 \mathrm{~Hz}$; Umino et al., 2018; Nomura et al., 2019). Response latencies depend also on the spatial patterns of stimuli as well as their contrast (Bolz et al., 1982; Baccus and Meister, 2002; Sagdullaev and McCall, 2005; Pearson and Kerschensteiner, 2015; Tikidji-Hamburyan et al., 2015). Here we used full-field stimuli at slightly lower light intensity level for in vivo recordings than for ex vivo ones (see Methods for details). Therefore, higher baseline activity and faster dynamics observed in awake animals cannot be simply attributed to the difference in the stimulus conditions.

Finally, when comparing retinal responses across different studies, one cannot ignore a possible effect of sampling bias because visual response dynamics are cell-type specific 421 (van Wyk et al., 2009; Krieger et al., 2017; Ravi et al., 2018; Tengölics et al., 2019). In an extreme case, delayed ON cells have the response latency slower than other RGC types by hundreds of milliseconds in an isolated mouse retina (Mani and Schwartz, 2017). Response 
dynamics are also species-specific. For example, ON RGCs show higher baseline activity and faster responses than OFF cells in the macaque (Chichilnisky and Kalmar, 2002) and guinea pig (Zaghloul et al., 2003) retinas, whereas slower in the salamander retina (Gollisch and Meister, 2008), and such asymmetry between ON and OFF dynamics is pathway-specific in the rat retina (Ravi et al., 2018). Moreover, kinetics can vary even for the same cell type, depending on the retinal location (Warwick et al., 2018) or light-adaptation state (Chang and He, 2014; Tengölics et al., 2019). Sampling bias can then skew the results in many different ways. In this study, however, we recorded a wide variety of cell types (Figure 2), and no apparent difference was found in the STA distributions across different recording conditions (Figure 5). We thus expect that the effects of sampling bias should be minimal on the observed differences between in vivo and ex vivo retinal response characteristics. between in vivo and ex vivo. This highlights the importance of studying retinal function in vivo, especially in the awake condition.

\section{Implications on retinal coding in vivo}

439 The optic nerve fiber forms an information bottleneck in the early visual system. The human 440 retina, for example, contains $\sim 10^{8}$ input neurons (photoreceptors) but only $\sim 10^{6}$ output neurons (RGCs) whose axons make up the optic nerve (Masland, 2012; Sanes and Masland, 2015). The retina is then expected to optimize the channel capacity by compressing the visual information as much as possible and conveying signals to the brain using as few spikes as possible (Attneave, 1954; Barlow and Rosenblith, 1961; Laughlin, 2001). Such an efficient coding hypothesis is well supported by both ex vivo experimental data and theoretical analyses. Under ex vivo conditions, RGCs are silent most of the time and fire spikes at high rates only when their selective stimulus features are presented (Gollisch and Meister, 2010; Baden et al., 2016; Jouty et al., 2018). This sparse RGC response, ensured by low 
efficiency and low redundancy in the visual representation of the retina (Doi et al., 2012). Furthermore, efficient coding principles can explain various physiological properties of the retina, such as separation of retinal outputs into multiple cell types (Gjorgjieva et al., 2019).

The retinal output characteristics we observed in vivo, however, provide a completely different view on the retinal code. First, unlike ex vivo conditions, RGCs in awake mice showed a high baseline activity with more linearized responses (Figures 3 and 6). Consequently, many awake ON cells primarily encoded light decrements by suppression from high baseline, rather than light increments by activation from low baseline. These cells convey the same information as conventional ON cells do—-the more spikes, the higher light intensity—but using much more spikes. Thus, the amount of information transmitted per spike is much lower in vivo than ex vivo, violating the efficient coding principles. Second, we found less variability in the visual response dynamics across different RGC types in vivo (Figure $5 \mathrm{E}-\mathrm{H}$ ). This suggests that temporal coding framework based on spike timing patterns may not be readily applicable in vivo. Latency coding, for example, will not work well in vivo as it requires noticeable differences in the response latencies between two or more channels, such as ON and OFF pathways converging onto ON-OFF RGCs (Gollisch and Meister, 2008). Our results thus favor dense rate coding in the retina, rather than sparse temporal coding.

What are the advantages of such energy-inefficient retinal coding? As shown in our awake recordings, one can gain faster response kinetics (Figure 5) and wider bandwidths (Figure 6). In addition, redundancy in the dense code helps transmit signals accurately even with intrinsically noisy spike trains. These features are all highly beneficial from a behavioral viewpoint-e.g., to detect predators robustly and promptly-and thus worth achieving for survival at an expense of energy cost. Interestingly, dense coding can be a general feature of early sensory processing, as suggested by high baseline firing in vestibular and cochlear nuclei (Fuchs and Kimm, 1975; Rhode and Smith, 1986; Warchol and Dallos, 1990; Beraneck and Cullen, 2007), tonic activity in hair cells (Sachs and Abbas, 1974; Wu et al., 2016), and dark current in vertebrate photoreceptors (Hagins et al., 1970; Okawa et al., 2008). In fact, the 
energy cost of dense retinal coding may not be so problematic with a relatively small number of neurons, as opposed to the total energy required for operating $\sim 10^{9}$ neurons in the cortex where sparse coding has certain advantages from the viewpoints of both energy efficiency and sensory processing (Olshausen and Field, 1996; Asari et al., 2006). Further characterizations of the retinal code in vivo will be needed to clarify what exactly the eye tells the brain.

\section{Effects of anesthetics in early visual system}

Anesthetics generally lower the excitability of nerve cells: e.g., isoflurane acts on the gammaaminobutyric acid (GABA) type A receptors to silence the brain (Jenkins et al., 1999). It thus makes sense that the retinal outputs were reduced under anesthesia (Figures 3 and 6) and showed slower dynamics (Figures 4 and 5) than in awake animals. It is, however, difficult to generalize the effects of anesthesia because multiple mechanisms of action are involved, exerting complex effects on the sensory systems in anesthetic- and dose-dependent manners (Populin, 2005). For example, while visual cortical responses are reduced under anesthesia (Haider et al., 2013; Vaiceliunaite et al., 2013), auditory responses are enhanced (Raz et al., 2014; Sellers et al., 2015) and the response latency becomes shorter in the auditory pathway (Ter-Mikaelian et al., 2007).

Our results are nevertheless consistent with previous studies on the effects of anesthesia in the early visual system. Specifically, anesthesia reduces the overall activity of both SC and dLGN neurons, and leads to multiple changes in temporal processing of these two major retinorecipient areas, such as reduced sensitivity to high temporal frequencies and longer response latencies (Zhao et al., 2014; Durand et al., 2016; De Franceschi and Solomon, 2018). While different anesthetics were used in these studies (urethane as opposed to isoflurane and FMM), our consistent findings suggest that such changes in the response dynamics arise largely from the retina. In contrast, the effects of anesthesia on spatial 


\section{Chronic recordings}

524 For implanting electrodes, animals were anesthetized (induction, 4\% isoflurane; maintenance,

dLGN (Durand et al., 2016), lower contrast sensitivity and larger receptive field sizes were reported in SC under urethane anesthesia (De Franceschi and Solomon, 2018). Moreover, SC neurons show increased orientation selectivity under isoflurane anesthesia (Kasai and Isa, 2021). Here we also found a larger fraction of motion sensitive cells under both isoflurane and FMM anesthesia (Figure 2D-F). Due to confounding effects of eye movements in awake animals, however, we were not able to fully analyze spatial response properties of retinal outputs in vivo. It is a future challenge to develop algorithms to compensate for the eye motion at a spatial resolution well below the RGC receptive field size (as small as 3 degrees; Zhang et al., 2012) for fully characterizing spatiotemporal processing of the retina in vivo.

\section{Materials and Methods}

No statistical method was used to predetermine the sample size. The significance level was 0.05 (with Bonferroni correction where appropriate) in all analyses unless noted otherwise. All experiments were performed under the license 233/2017-PR from the Italian Ministry of Health. The data analyses were done in Python.

\section{Animals}

A total of 43 female C57BL/6J mice were used (chronic, 3; acute, 23; failures, 17, including 10 initial unsuccessful attempts to record from the optic chiasm) at around three months of age at the time of the surgery. Mice were kept on a 12-h light / 12-h dark cycle and given water and food ad libitum. After the chronic implantation of electrodes, the animals were kept singlehoused.

$$
1-2 \% \text { ) and placed inside a stereotaxic apparatus (Neurostar). Throughout the surgery, }
$$
temperature was maintained at $37^{\circ} \mathrm{C}$ using a heating pad (Supertech Physiological), and eye ointment (VitA-POS) was used to prevent the eyes from drying. After positioning the mouse 
head, the scalp skin was disinfected (Betadine) and removed with scissors. Soft tissue was removed from the skull surface with a round scalpel, and ethanol and acetone were applied to the skull for disinfection and removal of any residual compounds. The skull was glazed with a drop of cyanoacrylate adhesive (Loctite 401) to secure in place the surrounding skin, and then registered in the stereotaxic controller software (NeuroStar). A dental drill (WPI) was used to leave three marks on the skull to label the entry point for targeting the optic tract: $[-1.34,+1.87$, $+4.74],[-1.70,+1.87,+4.74]$, and $[-1.82,+2.35,+4.07]$ in [Anterior-Posterior, Medial-Lateral, Dorsal-Ventral] coordinates, respectively. A hole was drilled for a reference silver wire (A-M Systems) above the cerebellum, and the wire was inserted sideways to avoid excessive brain damage. After covering the hole and wire with Vaseline, the wire was attached to the skull with cyanoacrylate adhesive and dental cement (Paladur, PALA). Subsequently, a customdesigned titanium head-plate was attached to the skull with dental cement, followed by a craniotomy (diameter, 1-2 mm) and durotomy. A chronic silicone probe (Buzsaki32L, NeuroNexus) was then inserted with the stereotaxic controller $(75 \mu \mathrm{m} / \mathrm{min})$ first to a depth of $2 \mathrm{~mm}$, retracted by $1 \mathrm{~mm}$ to release pressure created by the initial brain entry, and then to a depth of $4.5 \mathrm{~mm}$ from the skull surface. After reaching the desired depth, a microdrive (dDrive, NeuroNexus) was attached to the skull with dental cement, and the probe was removed from its mount and covered with a protective cap. The cables and connectors were cemented to the cap and covered with paraffin film (Bemis, Parafilm) to prevent the mouse from damaging the implant.

After the surgery, the animals were kept on a heating pad (Sera, Thermo comfort mat S) until they recovered from anesthesia. During the next four days, the mice received an antiinflammatory/antibiotic cocktail (Rimadyl/Baytril; $0.5 \mathrm{mg} / \mathrm{mL}$ each, $0.01 \mathrm{~mL} / \mathrm{g}$ ). The antibiotic (Baytril) was given for an additional three days through drinking water $(0.17 \mathrm{mg} / \mathrm{mL})$.

After a recovery period of five days, the mice were placed on a custom-made rotary treadmill (diameter, $20 \mathrm{~cm}$ ) with their head fixed for the recordings (at most two times a day, each for $<2$ hours). During the initial sessions, we moved the probe until visual responses 
555

556

557

were observed (Figure 1A-D). The electrophysiology data were recorded at $30 \mathrm{kHz}$ from each recording site (SmartBox, NeuroNexus) together with synchronization pulses from the visual stimulation device (see below). In total, we made 17 recording sessions from 3 out of 6 animals, where up to 10 cells were simultaneously recorded in each session $(4.4 \pm 2.4$ cells/session, mean \pm standard deviation).

After all the recording sessions, the electrode position was verified histologically (e.g., Figure 1E). The mice were anesthetized (2.5\% Avertin, $16 \mu \mathrm{L} / \mathrm{g}$, intraperitoneal injection) and perfused with paraformaldehyde (PFA; 4\% in phosphate buffer solution). The animal's head with the electrode left in position was collected without the skull base, and stored in fixative solution (4\% PFA) at $4^{\circ} \mathrm{C}$ for at least four days. This helped the brain tissue harden around the silicon probe, hence leaving a visible mark after removing the probe. Harvested brain tissue was then coronally sliced with a vibratome (Leica, VT1000S; thickness, $150 \mu \mathrm{m}$ ) and imaged under a bright-field microscope (Leica, LMD7000).

\section{Acute recordings}

Animals were anesthetized and placed inside a stereotaxic apparatus with a heating pad as described above. A contact lens (diameter, $3 \mathrm{~mm}$; Ocuscience) was used for the target eye to prevent it from drying. The scalp was removed, registered in the stereotaxic controller, and the three entry points for targeting the optic tract were marked with the stereotaxic drill. A well was made with dental cement around the marks later to hold saline solution on top of the brain. After a craniotomy (diameter, $2 \mathrm{~mm}$ ), an acute silicone probe (Buzsaki32L, Neuronexus) coated with a fluorescent dye (Dil stain, Invitrogen, D282) was slowly inserted into the brain $(100 \mu \mathrm{m} / \mathrm{min}$ ) with a micromanipulator (Sensapex, SMX) attached to the stereotaxic apparatus. While approaching the target depth, visual stimuli were presented (full-field contrast-inverting stimulus at $0.5 \mathrm{~Hz}$; see below). The probe was moved until a maximum number of visually responsive cells were seen at once (up to 20 cells; $7.7 \pm 5.3$ cells/session, mean \pm standard deviation; 52 sessions in total from 23 out of 27 animals). 
Throughout the recordings, the mouse was kept under anesthesia with $1 \%$ isoflurane.

The depth of anesthesia was monitored by the breathing rate $(\sim 1$ breath/s). Alternatively, we used a cocktail of fentanyl, medetomidine and midazolam (FMM) during the recordings. In this case, once the electrode was in position, an FMM solution (fentanyl, $0.05 \mathrm{mg} / \mathrm{kg}$; medetomidine, $0.5 \mathrm{mg} / \mathrm{kg}$; midazolam, $5 \mathrm{mg} / \mathrm{kg}$; in $0.9 \% \mathrm{NaCl}$ saline) was intraperitoneally administered, and the isoflurane dose was progressively decreased to $0 \%$. Buprenorphine $(0.1 \mathrm{mg} / \mathrm{kg})$ was injected 20 minutes after the termination of isoflurane, and the recording session was initiated 10 minutes after the buprenorphine injection. The depth of anesthesia was monitored through the heart rate (below around 300 beats per minute) and supplemental FMM anesthesia was provided when required. The electrophysiology data and the visual stimulation signals were recorded at $30 \mathrm{kHz} /$ electrode (SmartBox, NeuroNexus).

At the end of the recording session, the electrode location was verified histologically. After retracting the silicone probe, the animal was perfused as described above. The brain tissue was harvested and post-fixed overnight in $4 \%$ PFA at $4^{\circ} \mathrm{C}$. Coronal sections of the brain tissue (thickness, $150 \mu \mathrm{m}$ ) were then examined under a fluorescence microscope (Leica, LMD7000 with N2.1 filter cube) to visualize the trace left by the Dil stain on the probe.

\section{Visual stimulation}

Visual stimuli were presented by a custom gamma-corrected digital light processing (DLP) device (Texas Instruments, DLPDLCR3010EVM-LC) where the original green and red lightemitting diodes (LEDs) were replaced with ultra-violet (UV; $365 \mathrm{~nm}$; LZ1-00UV00, LED Engine) and infrared (IR; 950 nm; SFH 4725S, Osram) LEDs, respectively. The UV and blue $(465 \mathrm{~nm})$ lights were projected onto a spherical screen (radius, $20 \mathrm{~cm}$ ) with UV-reflective white paint (waterfowl store) placed $\sim 20 \mathrm{~cm}$ to the contralateral side of an animal's eye from the implanted probe (Figure 1A), whereas the IR light was used as synchronization pulses, recorded via a photodiode with a custom transimpedance amplifier. The visual stimuli (1280- 
eye position (Figure 1B). The maximum light intensity at the eye position was $15.4 \mathrm{~mW} / \mathrm{m}^{2}$ and $15.9 \mathrm{~mW} / \mathrm{m}^{2}$ for the UV and blue LEDs, respectively, leading to mesopic to photopic conditions. following set of visual stimuli: a randomly flickering full-field stimulus ( $5 \mathrm{~min} ; 60 \mathrm{~Hz}$ ), a moving grating stimulus (spatial frequencies of square waves, $3^{\circ}$ or $20^{\circ}$; moving speed, $7.5^{\circ} / \mathrm{s}$ or $15^{\circ} / \mathrm{s}$, in eight different directions), a full-field stimulus whose intensity followed a sinusoid $(1.5 \mathrm{~Hz})$ with a linearly increasing amplitude from $0 \%$ to $100 \%$ contrast over $10 \mathrm{~s}$ (10 trials; Figure 3), and a full-field sinusoidally flickering stimulus (maximum contrast) at different temporal frequencies $(1.875,3.75$ and $7.5 \mathrm{~Hz}$, each for $2 \mathrm{~s} ; 15 \mathrm{~Hz}$ for $1 \mathrm{~s} ; 10$ trials; Figure 4). The last two full-field stimuli were preceded by a sequence of "OFF-ON-OFF" stimulation at maximum contrast (full-field contrast inversion; $2 \mathrm{~s}$ each) and interleaved by a 1-s-long gray screen across trials. These stimuli were equivalent to those used for differentiating $\sim 30$ RGC types ex vivo (Baden et al., 2016; Jouty et al., 2018).

\section{Data analysis}

621 Spike sorting was performed with SpykingCircus (Yger et al., 2018) for semi-automatic cluster detection and Phy (Rossant, 2020) for data curation. In total, we obtained 75 cells for chronic recordings, and 298 and 103 cells for acute recordings under isoflurane and FMM anesthesia, respectively. Not all cells responded to the entire stimulus set, but cells responding to any of the presented stimuli were included in the analysis. For ex vivo recordings, we reanalyzed the data sets in Vlasiuk and Asari (2021). Specifically, the ex vivo data sets included the activity of 696 cells recorded with a multi-electrode array (from 18 isolated mouse retinas) in response to a randomly flickering full-field visual stimulus projected from a gamma-corrected cathoderay tube monitor (frame rate, $100 \mathrm{~Hz}$ ) or a DLP device $(60 \mathrm{~Hz})$. The maximum light intensity on the isolated retinas was $36 \mathrm{~mW} / \mathrm{m}^{2}$ (Vlasiuk and Asari, 2021). 


\section{Response quality}

632 We assessed the cell's response quality based on the trial-to-trial reliability of the response $633 r(t)$ during the "ON-OFF" period of the OFF-ON-OFF stimulus sequence (see Figure 2A for example). Specifically, the signal-to-noise ratio was calculated for each cell as follows:

$$
\operatorname{SNR}=\frac{\operatorname{var}[\langle r(t)\rangle]_{t}}{\left\langle\operatorname{var}[r(t)]_{t}\right\rangle}
$$

where $\langle\cdot\rangle$ indicates the mean over trials, and $\operatorname{var}[\cdot]_{t}$ the variance over time $t$ (bin size, $\Delta t=$

(Figure 2B).

\section{Response polarity}

640 To characterize the cell's preference to stimulus polarity, we defined an ON-OFF index using the responses to the full-field contrast inversion:

$$
\text { ON-OFF index }=\frac{r_{\mathrm{ON}}-r_{\mathrm{OFF}}}{r_{\mathrm{ON}}+r_{\mathrm{OFF}}}
$$

where $r_{\mathrm{ON}}$ and $r_{\mathrm{OFF}}$ are the mean firing rate during the $\mathrm{ON}$ and the second OFF periods of the OFF-ON-OFF stimulus sequence, respectively. Positive and negative ON-OFF index values indicate stronger responses to ON and OFF stimuli, respectively (Figure 2C).

\section{Motion sensitivity}

647 Direction-selectivity (DS) and orientation-selectivity (OS) indices were calculated by projecting 648 the responses to the moving grating stimuli onto a complex exponential:

$$
\text { DS or OS index }=\left\|\frac{\sum_{k} e^{-i \alpha \omega_{k}} \cdot r_{k}}{\sum_{k} r_{k}}\right\|
$$

where $\omega_{k}$ and $r_{k}$ are the angle of the $k$-th direction and the cell's corresponding responses, respectively; and $\alpha=1$ and 2 for the DS and OS indices, respectively. Cells were considered motion-sensitive when at least one of these indices was higher than 0.15 and $p<$ 


\section{Temporal frequency sensitivity}

Temporal frequency sensitivity was assessed by fitting an even power of sine function to the responses to the full-field sinusoidally flickering stimulus at different frequencies $h(1.875,3.75$, 7.5, or $15 \mathrm{~Hz}$; Figure 4):

$$
s(t)=A \cdot \sin ^{q}\left(\pi h t-\frac{\phi}{2}\right)+B
$$

where $A$ and $B \geq 0$ are the response amplitude and baseline activity, respectively; and $q=2 n$ with $n \in \mathbb{N}^{+}$is the exponent of the sine function. The phase $\phi \in[-\pi, \pi)$ indicates the relative position between the response peak and the sinusoidal stimulus patterns. For example, $\phi=$ $-\pi / 2$ and $\pi / 2$ are obtained if the response reaches its peak when the stimulus is brightest and darkest, respectively.

The fit quality was then assessed by the explained variance:

$$
R^{2}=1-\frac{\operatorname{var}[\text { Data }- \text { Fit }]}{\operatorname{var}[\text { Data }]}
$$

We set a threshold at 0.2 to select cells with a good fit hence responsive to the stimulus (Figure 4C), and compared the proportions of the responsive cells across different recording conditions (two-proportion z-test; Figure 4D).

\section{Contrast sensitivity}

We used a sigmoid-weighted sine-power function to characterize the responses to the flickering stimulus $(h=1.5 \mathrm{~Hz})$ with increasing contrast (Figure 3):

$$
S(t)=\frac{A}{1+e^{-\lambda\left(t-t_{0}\right)}} \cdot \sin ^{q}\left(\pi h t-\frac{\phi}{2}\right)+B
$$

where $t_{0}$ and $\lambda>0$ are the midpoint and the steepness of the sigmoid, respectively; and the other free parameters are the same as $s(t)$ in Eq.(4). The fitted parameter values were then compared across different recording conditions (Mann-Whitney $U$-test for the baseline $B$, 
3F). As a measure of contrast sensitivity, we used an estimated response magnitude at $10 \%$ contrast from Eq.(6).

\section{Temporal filters and static nonlinear gain functions}

680 For systematically characterizing the response dynamics, we used stimulus ensemble statistical techniques ("reverse correlation" methods; 500 ms window) to calculate the linear filter (Figure 5) and static nonlinear gain function (Figure 6) of the recorded cells in response to a randomly flickering visual stimulus (Meister et al., 1994; Chichilnisky, 2001). First, we obtained the linear filter of each cell by calculating a spike-triggered average (STA) of the stimulus with \pm 1 being "white" and "black," respectively. As a quality measure, $p$-value was computed for each time bin against a null hypothesis that the STA follows a normal distribution with a mean of zero and a variance of $1 / C$, where $C$ is the total number of spikes. As a measure of the cell's temporal frequency tuning, we then estimated the peak latency by fitting a difference-of-Gaussian curve to the linear filter (e.g., Figure 5A); and the spectral peak frequency by the Fourier analysis on the fitted curve (e.g., Figure 5B). The curve fitting quality was assessed by the explained variance $R^{2}$ as in Eq. (5). We discarded the cells if $p>10^{-18}$ for all time bins or $R^{2}<0.8$. We ran a $t$-test to compare the temporal frequency tuning properties at the population level across recording conditions (Figure 5C,D) or across different cell types in each recording condition (Figure 5E-H). Spatial response properties were not examined in this study because complex nonlinear kinematics of eye movements in the awake condition precluded the analysis at a high enough spatial resolution; and because it has been shown that anesthesia has no effect on the spatial processing in dLGN, a direct downstream of the retina (Durand et al., 2016). $N$ (stimulus|response) projected onto the L2-normalized STA (bin size, 0.1 ) and that of the entire stimulus ensembles $N$ (stimulus) (e.g., Figure 6B): 


$$
P(\text { response } \mid \text { stimulus })=\frac{N(\text { stimulus } \mid \text { response })}{N(\text { stimulus })} / \Delta t
$$

A sigmoid function was fitted to $P$ (response|stimulus) for smoothing. The neutral stimulus response was then defined as the vertical-intercept of the sigmoid function at the horizontal axis value of zero: i.e., the firing rate when the stimulus is orthogonal to the STA. We ran a $t$ test to compare the neutral stimulus responses and the average firing rates during the stimulus presentation at the population level across conditions (Figure 6A,C); and Mann-Whitney $U$ test to compare the midpoint of the sigmoid (Figure $6 \mathrm{H})$.

\section{Cell-type classification}

711

In this study, we were not able to perform a morphological analysis of individual cells as we employed blind in vivo recording methods (Figure 1). We thus focused on the visual response properties for classifying cell-types in the following two ways.

First, we classified cells in vivo using the response quality, response polarity, and motion sensitivity (Figure 2). Specifically, we first divided the cells into two groups: reliably responsive ones with SNR $>0.15$ (Eq. (2)) and the other low quality ones ("N/A" type; Figure 2B). We then set thresholds at \pm 0.25 for the ON-OFF index (Eq. (3)) to identify ON, OFF, and ON/OFF types within the reliably responsive cells that increased firing in response to light increments, light decrements, and both, respectively (Figure 2C). For some cells where these measures were not available, we used the responses to the full-field sinusoidally flickering stimuli to calculate the response quality and polarity in a similar manner. Within the ON cells, we further identified an "OFF-suppressive" type if they had a significant negative amplitude in Eq. (6) with $95 \%$ confidence intervals in the parameter estimation. Independently, we also labeled cells as motion sensitive or not, based on the DS/OS indices as described above (Eq.(3); Figure 2D-F).

Second, because these measures are not available for the ex vivo data sets (Vlasiuk and Asari, 2021), we performed cell-type classification using the temporal filter properties to make a fair comparison between the ex vivo and in vivo data sets (Figures 5 and 6). 
Specifically, we first ran a principal component analysis (PCA) on the temporal filters (L2normalized STA) obtained in each condition (Gollisch and Meister, 2008; Asari and Meister, 2014). The first two principal components (PC1, monophasic filter; and PC2, biphasic filter) were largely sufficient to fit all the temporal filter dynamics, accounting for $78-86 \%$ of the total variance in each condition. Each temporal filter is a point in the PCA biplot (i.e., the twodimensional space spanned by PC1 and PC2), and we grouped its shape into four subtypes based on its position: monophasic OFF, biphasic OFF, monophasic ON, and biphasic ON from the first to the fourth quadrants, respectively.

\section{Acknowledgments}

This work was supported by research grants from EMBL (H.A.). The EMBL Histology Facility and the Advanced Light Microscopy Facility are acknowledged for support in sample preparation and image acquisition for histological analyses, respectively. EMBL IT Support is acknowledged for provision of computer and data storage servers. We thank Dmitry Molotkov for his help in setting up the recording rig, and all the Asari lab members as well as Cornelius Gross and Santiago Rompani for many useful discussions.

\section{References}

Ames, A., Nesbett, F. B. (1981). In Vitro Retina as an Experimental Model of the Central Nervous System. J Neurochem, 37(4), 867-877.

Asari, H., Meister, M. (2014). The projective field of retinal bipolar cells and its modulation by visual context. Neuron, 81(3), 641-652.

Asari, H., Pearlmutter, B. A., Zador, A. M. (2006). Sparse representations for the cocktail party problem. J Neurosci, 26(28), 7477-7490.

Atick, J. J., Redlich, A. N. (1990). Towards a Theory of Early Visual Processing. Neural Comput, 2(3), 308-320. 
Attneave, F. (1954). Some informational aspects of visual perception. Psychol Rev, 61(3), 183-193.

Baccus, S. A., Meister, M. (2002). Fast and slow contrast adaptation in retinal circuitry. Neuron, 36(5), 909-919. functional diversity of retinal ganglion cells in the mouse. Nature, 529(7586), 345-350. selectively to direction and speed of image motion in the rabbit. J Physiol, 173(3), 377-407. of sensory messages. In Sensory Communication (pp. 217-234). MIT Press. vestibular and optokinetic stimulation in the alert mouse. J Neurophysiol, 98(3), 1549-1565. of single lateral geniculate cells. J Physiol (Lond), 162, 451-472. transient $(\mathrm{Y})$ cells in the cat retina. J Physiol, 328, 171-190.

Chang, L., He, S. (2014). Light adaptation increases response latency of alpha ganglion cells via a threshold-like nonlinearity. Neuroscience, 256, 101-116. sensory representations in the non-lemniscal mouse inferior colliculus. Commun Biol, 2, 356. 
Chichilnisky, E. J., Kalmar, R. S. (2002). Functional asymmetries in ON and OFF ganglion cells of primate retina. J Neurosci, 22(7), 2737-2747.

De Franceschi, G., Solomon, S. G. (2018). Visual response properties of neurons in the superficial layers of the superior colliculus of awake mouse. J Physiol, 596(24), 6307-6332.

Doi, E., Gauthier, J. L., Field, G. D., Shlens, J., Sher, A., Greschner, M., Machado, T. A., Jepson, L. H., Mathieson, K., Gunning, D. E., Litke, A. M., Paninski, L., Chichilnisky, E. J., Simoncelli, E. P. (2012). Efficient Coding of Spatial Information in the Primate Retina. J Neurosci, 32(46), 16256-16264.

Dombeck, D. A., Khabbaz, A. N., Collman, F., Adelman, T. L., Tank, D. W. (2007). Imaging large-scale neural activity with cellular resolution in awake, mobile mice. Neuron, 56(1), 43-57.

Durand, S., Iyer, R., Mizuseki, K., de Vries, S., Mihalas, S., Reid, R. C. (2016). A Comparison of Visual Response Properties in the Lateral Geniculate Nucleus and Primary Visual Cortex of Awake and Anesthetized Mice. J Neurosci, 36(48), 12133-12156.

Enroth-Cugell, C., Robson, J. G. (1966). The contrast sensitivity of retinal ganglion cells of the cat. J Physiol, 187, 517-552.

Esposti, F., Johnston, J., Rosa, J. M., Leung, K.-M., Lagnado, L. (2013). Olfactory Stimulation Selectively Modulates the OFF Pathway in the Retina of Zebrafish. Neuron, 79(1), 97-110.

Franke, K., Chagas, A. M., Zhao, Z., Zimmermann, M. J., Bartel, P., Qiu, Y., Szatko, K. P., Baden, T., Euler, T. (2019). An arbitrary-spectrum spatial visual stimulator for vision research. Elife, 8, e48779.

Fuchs, A. F., Kimm, J. (1975). Unit activity in vestibular nucleus of the alert monkey during horizontal angular acceleration and eye movement. J Neurophysiol, 38(5), 1140-1161. 
800

801

802

803

804

805

806

807

808

809

810

811

812

813

814

815

Geng, Y., Dubra, A., Yin, L., Merigan, W. H., Sharma, R., Libby, R. T., Williams, D. R. (2012). Adaptive optics retinal imaging in the living mouse eye. Biomed Opt Express, 3(4), 715-734.

Gjorgjieva, J., Meister, M., Sompolinsky, H. (2019). Functional diversity among sensory neurons from efficient coding principles. PLoS Comput Biol, 15(11), e1007476.

Gollisch, T., Meister, M. (2008). Rapid neural coding in the retina with relative spike latencies. Science, 319(5866), 1108-1111.

Gollisch, T., Meister, M. (2010). Eye Smarter than Scientists Believed: Neural Computations in Circuits of the Retina. Neuron, 65, 150-164.

Greenberg, D. S., Houweling, A. R., Kerr, J. N. D. (2008). Population imaging of ongoing neuronal activity in the visual cortex of awake rats. Nat Neurosci, 11(7), 749-751.

Hagins, W. A., Penn, R. D., Yoshikami, S. (1970). Dark Current and Photocurrent in Retinal Rods. Biophys J, 10(5), 380-412.

Haider, B., Häusser, M., Carandini, M. (2013). Inhibition dominates sensory responses in the awake cortex. Nature, 493(7430), 97-100.

Hartline, H. K. (1938). The response of single optic nerve fibers of the vertebrate eye to illumination of the retina. Am J Physiol, 121, 400-415.

Hong, G., Fu, T.-M., Qiao, M., Viveros, R. D., Yang, X., Zhou, T., Lee, J. M., Park, H.-G., Sanes, J. R. (2018). A method for single-neuron chronic recording from the retina in awake mice. Science, 360(6396), 1447-1451.

Jacoby, J., Schwartz, G. W. (2018). Typology and Circuitry of Suppressed-by-Contrast Retinal Ganglion Cells. Front Cell Neurosci, 12, 269.

Jenkins, A., Franks, N. P., Lieb, W. R. (1999). Effects of temperature and volatile anesthetics on GABA(A) receptors. Anesthesiology, 90(2), 484-491. 
Jouty, J., Hilgen, G., Sernagor, E., Hennig, M. H. (2018). Non-parametric Physiological Classification of Retinal Ganglion Cells in the Mouse Retina. Front Cell Neurosci, 12, 481. Jun, J. J., Steinmetz, N. A., Siegle, J. H., Denman, D. J., Bauza, M., Barbarits, B., Lee, A. K., Anastassiou, C. A., Andrei, A., Aydın, Ç., Barbic, M., Blanche, T. J., Bonin, V., Couto, J., Dutta, B., Gratiy, S. L., Gutnisky, D. A., Häusser, M., Karsh, B., .. Harris, T. D. (2017). Fully Integrated Silicon Probes for High-Density Recording of Neural Activity. Nature, 551(7679), 232-236.

Kaplan, E., Shapley, R. (1984). The origin of the S (slow) potential in the mammalian lateral geniculate nucleus. Exp Brain Res, 55(1), 111-116.

Kasai, M., Isa, T. (2021). Effects of light isoflurane anesthesia on organization of direction and orientation selectivity in the superficial layer of the mouse superior colliculus. $\mathrm{J}$ Neurosci, JN-RM-1196-21. DOI: 10.1523/JNEUROSCI.1196-21.2021 optomotor and optokinetic reflexes in mice. J Neurophysiol, 118(1), 300-316.

Krieger, B., Qiao, M., Rousso, D. L., Sanes, J. R., Meister, M. (2017). Four alpha ganglion cell types in mouse retina: Function, structure, and molecular signatures. PLoS One, 12(7), information. Curr Opin Neurobiol, 11(4), 475-480. 
Li, J. Y., Schmidt, T. M. (2018). Divergent projection patterns of M1 ipRGC subtypes. J Comp Neurol, 526(13), 2010-2018.

Liang, L., Fratzl, A., Goldey, G., Ramesh, R. N., Sugden, A. U., Morgan, J. L., Chen, C., Andermann, M. L. (2018). A Fine-Scale Functional Logic to Convergence from Retina to Thalamus. Cell, 173(6), 1343-1355.e24. 3934.e9.

Mani, A., Schwartz, G. W. (2017). Circuit Mechanisms of a Retinal Ganglion Cell with Stimulus-Dependent Response Latency and Activation Beyond Its Dendrites. Curr Biol, 27(4), 471-482. active inputs to X-and Y-cells. J Neurophysiol, 49(2), 303-324.

Mastronarde, D. N. (1985). Two types of cat retinal ganglion cells that are suppressed by contrast. Vision Res, 25(9), 1195-1196.

Meister, M., Pine, J., Baylor, D. A. (1994). Multi-neuronal signals from the retina: acquisition and analysis. J Neurosci Methods, 51(1), 95-106.

Niell, C. M., Stryker, M. P. (2010). Modulation of visual responses by behavioral state in mouse visual cortex. Neuron, 65(4), 472-479. measurement methods using a touchscreen-based visual temporal discrimination task in the behaving mouse. Neurosci Res, 148, 28-33. 

Mammalian Rod Photoreceptors in Darkness and in Light. Curr Biol, 18(24), 1917-1921. by learning a sparse code for natural images. Nature, 381(6538), 607-609.

Pearson, J. T., Kerschensteiner, D. (2015). Ambient illumination switches contrast preference of specific retinal processing streams. J Neurophysiol, 114(1), 540-550.

Pitkow, X., Meister, M. (2012). Decorrelation and efficient coding by retinal ganglion cells. Nat Neurosci, 15(4), 628-635. (2006). The centrifugal visual system of vertebrates: a comparative analysis of its functional anatomical organization. Brain Res Rev, 52(1), 1-57. cochlear nucleus of the cat. J Neurophysiol, 56(2), 261-286.

Rossant, C. (2020). Phy: interactive visualization and manual spike sorting of large-scale ephys data. GitHub. https://github.com/cortex-lab/phy

Sachs, M. B., Abbas, P. J. (1974). Rate versus level functions for auditory-nerve fibers in cats: tone-burst stimuli. J Acoust Soc Am, 56(6), 1835-1847. 
Sagdullaev, B. T., McCall, M. A. (2005). Stimulus size and intensity alter fundamental receptive-field properties of mouse retinal ganglion cells in vivo. Vis Neurosci, 22(5), 649659.

Sanes, J. R., Masland, R. H. (2015). The types of retinal ganglion cells: current status and implications for neuronal classification. Annu Rev Neurosci, 38, 221-246.

Schröder, S., Steinmetz, N. A., Krumin, M., Pachitariu, M., Rizzi, M., Lagnado, L., Harris, K. D., Carandini, M. (2020). Arousal Modulates Retinal Output. Neuron, 107(3), 487-495. Sellers, K. K., Bennett, D. V., Hutt, A., Williams, J. H., Fröhlich, F. (2015). Awake vs. anesthetized: layer-specific sensory processing in visual cortex and functional connectivity between cortical areas. J Neurophysiol, 113(10), 3798-3815.

Shekhar, K., Lapan, S. W., Whitney, I. E., Tran, N. M., Macosko, E. Z., Kowalczyk, M., Adiconis, X., Levin, J. Z., Nemesh, J., Goldman, M., McCarroll, S. A., Cepko, C. L., Regev, A., Sanes, J. R. (2016). Comprehensive Classification of Retinal Bipolar Neurons by Single-Cell Transcriptomics. Cell, 166(5), 1308-1323.e30.

Sibille, J., Gehr, C., Benichov, J. I., Balasubramanian, H., Teh, K. L., Lupashina, T., Vallentin, D., Kremkow, J. (2021). Strong and specific connections between retinal axon mosaics and midbrain neurons revealed by large scale paired recordings. bioRxiv. 10.1101/2021.09.09.459396

Strauss, O. (2005). The retinal pigment epithelium in visual function. Physiol Rev, 85(3), 845-881.

Tabata, H., Shimizu, N., Wada, Y., Miura, K., Kawano, K. (2010). Initiation of the optokinetic response (OKR) in mice. J Vis, 10(1), 1-17.

Tengölics, Á. J., Szarka, G., Ganczer, A., Szabó-Meleg, E., Nyitrai, M., Kovács-Öller, T., Völgyi, B. (2019). Response Latency Tuning by Retinal Circuits Modulates Signal Efficiency. Sci Rep, 9(1), 15110. 

properties between auditory midbrain and cortex in the awake Mongolian gerbil. J Neurosci, 27(23), 6091-6102.

Tien, N.-W., Pearson, J. T., Heller, C. R., Demas, J., Daniel, K. (2015). Genetically Identified Suppressed-by-Contrast Retinal Ganglion Cells Reliably Signal Self-Generated Visual Stimuli. J Neurosci, 35(30), 10815-10820. every change in ambient illuminance. Nat Neurosci, 18(1), 66-74.

Umino, Y., Pasquale, R., Solessio, E. (2018). Visual Temporal Contrast Sensitivity in the Behaving Mouse Shares Fundamental Properties with Human Psychophysics. eNeuro, 5(4), ENEURO.0181-18.2018. in mouse primary visual cortex. J Neurophysiol, 110(4), 964-972.

van Wyk, M., Wässle, H., Taylor, W. R. (2009). Receptive field properties of ON- and OFFganglion cells in the mouse retina. Vis Neurosci, 26(3), 297-308.

Vidal-Sanz, M., Galindo-Romero, C., Valiente-Soriano, F. J., Nadal-Nicolás, F. M., OrtinHypertension. Front Neurosci, 11, 235. One, 16(7), e0254611.

Wang, Y. V., Weick, M., Demb, J. B. (2011). Spectral and temporal sensitivity of conemediated responses in mouse retinal ganglion cells. J Neurosci, 31(21), 7670-7681. 
Warchol, M. E., Dallos, P. (1990). Neural coding in the chick cochlear nucleus. J Comp Physiol A, 166(5), 721-734.

Warwick, R. A., Kaushansky, N., Sarid, N., Golan, A., Rivlin-Etzion, M. (2018). Inhomogeneous Encoding of the Visual Field in the Mouse Retina. Curr Biol, 28(5), 655665.e3.

Weyand, T. G. (2007). Retinogeniculate transmission in wakefulness. J Neurophysiol, 98(2), 769-785. cortical calcium dynamics in anesthetized and awake mice. PLoS One, 12(10), e0185759.

Wu, J. S., Young, E. D., Glowatzki, E. (2016). Maturation of Spontaneous Firing Properties after Hearing Onset in Rat Auditory Nerve Fibers: Spontaneous Rates, Refractoriness, and Interfiber Correlations. J Neurosci, 36(41), 10584-10597.

Yan, W., Laboulaye, M. A., Tran, N. M., Whitney, I. E., Benhar, I., Sanes, J. R. (2020). Mouse Retinal Cell Atlas: Molecular Identification of over Sixty Amacrine Cell Types. J Neurosci, 40(27), 5177-5195.

Yger, P., Spampinato, G. L., Esposito, E., Lefebvre, B., Deny, S., Gardella, C., Stimberg, M., Jetter, F., Zeck, G., Picaud, S., Duebel, J., Marre, O. (2018). A spike sorting toolbox for up to thousands of electrodes validated with ground truth recordings in vitro and in vivo. Elife, 7, e34518. 

R., Merigan, W. H. (2014). Imaging light responses of foveal ganglion cells in the living macaque eye. J Neurosci, 34(19), 6596-6605. ganglion cells cause different contrast sensitivities. J Neurosci, 23(7), 2645-2654. type of the mouse retina is a selective feature detector. Proc Natl Acad Sci U S A, 109(36), E2391-E2398. selectivity of looming-evoked responses in the superior colliculus of awake mice. Neuron, 84(1), 202-213. latency code for interaural phase difference discrimination in the guinea pig inferior colliculus. J Neurosci, 31(25), 9192-9204. 